Review paper

\title{
Functional lung imaging with synchrotron radiation: Methods and preclinical applications
}

\author{
Sam Bayat ${ }^{\mathrm{a}, \mathrm{b}, \text {, }}$, Liisa Porra ${ }^{\mathrm{c}, \mathrm{d}}$, Pekka Suortti ${ }^{\mathrm{c}}$, William Thomlinson ${ }^{\mathrm{c}, \mathrm{e}}$ \\ ${ }^{a}$ Grenoble University Hospital, Department of Pulmonology and Clinical Physiology, France \\ ${ }^{\mathrm{b}}$ Synchrotron Radiation for Biomedicine (STROBE) Laboratory, INSERM UA7, University of Grenoble Alps, Grenoble, France \\ ${ }^{c}$ Department of Physics, University of Helsinki, Helsinki, Finland \\ ${ }^{\mathrm{d}}$ Helsinki University Hospital, Helsinki, Finland \\ e Department of Physics, University of Saskatchewan, Saskatoon, Canada
}

\section{A R T I C L E I N F O}

\section{Keywords:}

Respiratory distress syndrome, Adult

Ventilator-induced lung injury

Mechanical ventilation

Pulmonary alveoli

Tomography, X-ray Computed

Synchrotrons

Phase-contrast imaging

\begin{abstract}
A B S T R A C T
Many lung disease processes are characterized by structural and functional heterogeneity that is not directly appreciable with traditional physiological measurements. Experimental methods and lung function modeling to study regional lung function are crucial for better understanding of disease mechanisms and for targeting treatment. Synchrotron radiation offers useful properties to this end: coherence, utilized in phase-contrast imaging, and high flux and a wide energy spectrum which allow the selection of very narrow energy bands of radiation, thus allowing imaging at very specific energies. K-edge subtraction imaging (KES) has thus been developed at synchrotrons for both human and small animal imaging. The unique properties of synchrotron radiation extend X-ray computed tomography (CT) capabilities to quantitatively assess lung morphology, and also to map regional lung ventilation, perfusion, inflammation and biomechanical properties, with microscopic spatial resolution. Four-dimensional imaging, allows the investigation of the dynamics of regional lung functional parameters simultaneously with structural deformation of the lung as a function of time. This review summarizes synchrotron radiation imaging methods and overviews examples of its application in the study of disease mechanisms in preclinical animal models, as well as the potential for clinical translation both through the knowledge gained using these techniques and transfer of imaging technology to laboratory X-ray sources.
\end{abstract}

\section{Introduction}

The lung is a complex organ that is one of the primary ports of entry to the body of pollutants and micro-organisms but also a route for therapeutic aerosol administration. Many lung disease processes are characterized by structural and functional heterogeneity that is not directly measurable with traditional physiological measurements. Most of what is known about lung and airway function is based on traditional methods such as spirometry and plethysmography that measure overall functional parameters. However, it is known that both normal and pathological lung function, in chronic diseases such as asthma, chronic obstructive lung disease (COPD), or interstitial lung diseases are heterogeneous. Experimental methods to image regional lung function are crucial for better understanding of disease mechanisms and for targeting treatment. An ideal technique for lung function imaging should have both high spatial and temporal resolution, allow for quantitative measurements of functional parameters and provide the ability to image the underlying lung morphology. Four-dimensional (4D) imaging, in which high-resolution mapping of lung functional parameters is recorded simultaneously with structural deformation of tissue morphology as a function of time, provides the basis for comprehensive modeling of the regional dynamics of lung function. Recently, impressive results toward this goal have been achieved using synchrotron radiation sources, as described in this review.

Lung morphology has been described in great detail [1]. Sections through lung casts give high resolution 2D and 3D images of the airways and acini. These images are stationary, but they provide boundary conditions for modeling of dynamic processes, such as the distribution of ventilation, perfusion, and aerosol deposition. The bronchial tree is to a certain degree a self-similar or fractal structure. Each bifurcation is slightly asymmetrical, which leads to an asymmetrical statistical distribution of ventilation and other determinants of lung function $[2,3]$.

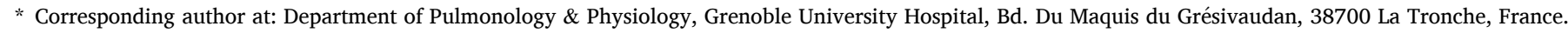

E-mail address: sbayat@chu-grenoble.fr (S. Bayat).
} 
At present, there are significant limitations in lung imaging arising from low available X-ray flux, large source size, detector performance and radiation damage to tissues. In this review new imaging modalities based on the use of synchrotron radiation are presented. In particular, the coherence of the radiation is utilized in phase-contrast imaging. There are several methods where the distortion of the X-ray wave front in the object leads to strong edge enhancement within the images due to interference of the transmitted and refracted radiation. In weakly attenuating tissues such as the lung, refraction can be orders of magnitude greater than absorption, particularly at higher energies. With the application of "phase retrieval" algorithms, images of strongly increased contrast in lung tissue are obtained, while the X-ray dose may be reduced in proportion. The propagation-based imaging technique (PBI) has proven to be especially suited for lung imaging due to sharp interference fringes caused by the air-to-tissue interfaces in the lung microstructures $[4,5]$.

Conventional clinical X-ray imaging, based on absorption contrast, is limited specifically by the low flux available in standard X-ray imaging systems. Synchrotrons have the advantage of generating very high flux radiation. This allows the selection of very narrow energy bands from the full radiation spectrum for imaging at very specific energies.

In this review we present several synchrotron radiation-based imaging modalities, and their use in preclinical animal models of lung disease. These methods include: (i) free propagation-based phasecontrast lung imaging (PBI) [6,7]; (ii) speckle-based lung imaging [8,9]; (iii) 4D lung imaging $[10,11]$, and (iv) K-edge subtraction (KES) imaging [12]. These methods have been used to investigate the regional function of normal lung as well as the manifestations of lung diseases, or the effect of pharmacological agents and interventions.

\section{Properties of synchrotron radiation and X-ray optics}

When a charged particle moves in an accelerating field it emits electromagnetic radiation. In a circular accelerator the electron is subjected to centripetal acceleration. At low velocities the emission pattern is a "doughnut" with the axis pointing to the center of the orbit. When the velocity approaches the light velocity, $c$, the distribution collapses to a narrow cone in the forward direction. The angular opening of the cone is about $1 / \gamma=m c^{2} / E$, where $m$ is the electron rest mass and $E$ the total energy. In a high-energy accelerator, $\gamma$ is of the order of $10^{4}$ so that the cone opening is $\sim 0.1 \mathrm{mrad}$.

A modern synchrotron source is a vacuum tube polygon where the electrons are guided by dipole bending magnets and focusing magnets. The circumference is several hundred meters, and the intensity of radiation is enhanced in the straight sections where magnet arrays make the electron beam wiggle or undulate. The electrons move in bunches that are a few mm long and a few tens of micrometers in diameter. The fill pattern can be varied according to the needs of the experiment, from a single bunch to hundreds of equally spaced bunches. The electron path in a wiggler or undulator is approximately sinusoidal, and the radiation cones add up in the forward direction. In a wiggler the beam is an incoherent sum of the individual cones within a horizontal fan. In an undulator the constructive interference produces narrow bands of harmonic wavelengths in the central cone.

The synchrotron radiation beam exits the storage ring through the front end and an opening in the shield wall. The power of the beam is many orders of magnitude larger than the power from a conventional Xray tube. For instance, the total power of the wiggler beam at the Medical Beamline of the European Synchrotron Radiation Facility (ESRF) is about $15 \mathrm{~kW}$, i.e. $10^{5}$ times the power of the beam from a rotating anode X-ray tube. Due to its unique properties, the use of synchrotron radiation allows many applications outside the reach of conventional X-ray sources and methods.

The wiggler or undulator beam is further conditioned by slits, filters and X-ray optical components. Monochromators, usually made of perfect silicon crystals, are used to separate a wavelength band from the continuous wiggler radiation or to separate a harmonic from the undulator spectrum. Focusing elements include bent crystals, multilayer structures, mirrors and compound refractive lenses. Due to the high heat load of the beam there are many technological challenges in the actual beamline design.

\section{Imaging modalities}

\subsection{Phase and absorption contrast}

In recent years, the principles of X-ray imaging for medical applications have been discussed in several reviews [7,13-15]. X-rays interact with the electrons of the target leading to various excitations in the target and scattering of the rays. Ignoring collective phenomena, the scattering can be elastic, inelastic (Compton) and resonant scattering. Scattering distributions carry information about the structures and electronic states of the object and are useful for medical imaging.

Image contrast is often due to interference within the beam that has traversed the object, so that a certain degree of coherence is needed. The exit beam is a sum of the direct beam and the scattered beam of amplitude $A=r_{\mathrm{e}} f$, where $r_{\mathrm{e}}=e^{2} / m c^{2}=2.82 \times 10^{-13} \mathrm{~cm}$ is the free electron scattering amplitude (electron classical radius). There is a phase shift in scattering, so that the amplitude is a complex number, $f=f_{0}+f^{\prime}$ $+\mathrm{i} f$ ". In the following, only forward scattering is considered, so $f_{0}=\mathrm{Z}$, the number of electrons of the atom. The refractive index of the object is:

$n=1-\left(N \lambda^{2} / 2 \pi\right) r_{e} f=1-\delta-i \beta$

where $N$ is the number of atoms per unit volume, $\lambda=2 \pi / k$ is the X-ray wavelength, $\delta$ is the phase shift term and $\beta$ is the absorption term. The coefficient of change of phase $\varphi$ is given by

$\eta(x, y, z)=\delta k=\mathrm{N} \lambda r_{e}\left(Z+f^{\prime}\right),=r_{e} \lambda \mathrm{N}_{\mathrm{A}} \mathrm{Z} \rho(x, y, z) / M$

where $N_{\mathrm{A}}$ is Avogadro's number and $M$ the atomic weight. Since $M / Z$ is nearly 2 for light elements,

$\delta=1.35 \times 10^{-4} \lambda^{2}\left[\mathrm{~nm}^{2}\right] \rho\left[\mathrm{gcm}^{-3}\right]$

The linear photoelectric absorption coefficient (relative loss of intensity per unit path length) is

$\sigma_{\mathrm{ph}}=2 N \lambda r_{\mathrm{e}} f^{\prime \prime}$

The coefficients of phase change and absorption have very different dependencies on the X-ray Energy $E$. At high energies $\eta$ is proportional to $1 / E$, while above the $K$-absorption edge $\sigma_{\mathrm{ph}}$ is proportional to $1 / E^{3}$. The total attenuation coefficient $\mu$ is the sum of the photoelectric absorption coefficient and the elastic (coherent) and inelastic (incoherent) scattering coefficients. It is important to note that at high energies Compton scattering dominates the X-ray attenuation.

The numerical values of $\eta$ and $\mu$ are very different at energies used for biomedical imaging. For the main constituents of soft tissue, $\eta$ is more than two orders of magnitude larger than $\mu$. There is a fundamental advantage in phase-contrast imaging with respect to absorption-contrast imaging in addition to the favorable $\eta / \mu$ ratio. The signal in absorptioncontrast imaging is the reduction of transmitted intensity due to actual absorption and all scattering that does not reach the detector. This can result in small variations in a large quantity. In phase-contrast imaging, the particular signal of forward scattering is extracted, while other signals remain in the background.

A lateral gradient of the real part $\delta$ of $(1-n)$ causes a deviation of the $\mathrm{X}$-ray beam. The refraction angle $\varepsilon$ is calculated from Snell's law at the interface. If the propagation direction is $\mathrm{z}$ and the deviation is in the $\mathrm{y}$ direction:

$\varepsilon=\Delta \delta \tan \alpha=(\lambda / 2 \pi)(\mathrm{d} \varphi / \mathrm{dy})$

Here $\Delta \delta$ is the change of the refractive index, and $\alpha$ is the angle 
between the normal of the interface and the incident ray. Sharp changes in propagation take place when the ray is (nearly) parallel to the interface. This is illustrated in Fig. 1, where the beam is refracted in an idealized pure phase object of no absorption (Fig. 1a). Imaging requires solving the phase from the intensity of the transmitted beam. The methods fall in three categories: (i) interferometry, where the phase is solved directly by comparison to the coherent reference beam [16,17], (ii) grating interferometry (GI) or analyzer-based imaging (ABI), where the refraction angle is measured (Fig. 1c) [9], and (iii) free propagation image of the second derivative (Fig. 1b) [18]. As shown in Fig. 1, at the edges the refracted beam is in-phase or out-of-phase with the direct beam, depending on the sign of the phase gradient ( $\mathrm{d} \varphi / \mathrm{dy})$. After a sufficient distance of free propagation, the intensity is that of the second derivative of the phase object.

The ultimate goal of phase-contrast imaging is 3D reconstruction of the refractive index decrement $\delta(x, y, z)$, and from that, the density variations of the object. Setting aside crystal interferometry, which is a demanding method for medical imaging, this means extracting the phase gradient from the refraction angle distribution or phase retrieval from the Laplacian. It is important that the angle $\alpha$ at the exit is the sum of deviations along the beam path, so that CT reconstruction of the differential phase is possible from the projections of the refraction angle using standard algorithms. On the other hand, in the propagation imaging the projected phase distribution must be retrieved from the Laplacian term of the intensity distribution for CT reconstruction of phase $\varphi$.

Scattering is considered a nuisance in absorption-based imaging because it gives rise to a diffuse background. Phase reconstruction from the transmitted intensity distribution is the primary objective in phasecontrast imaging, but scattering away from the beam carries important information, which can be extracted by different imaging methods. The effects of scattering are observed as increased attenuation of the

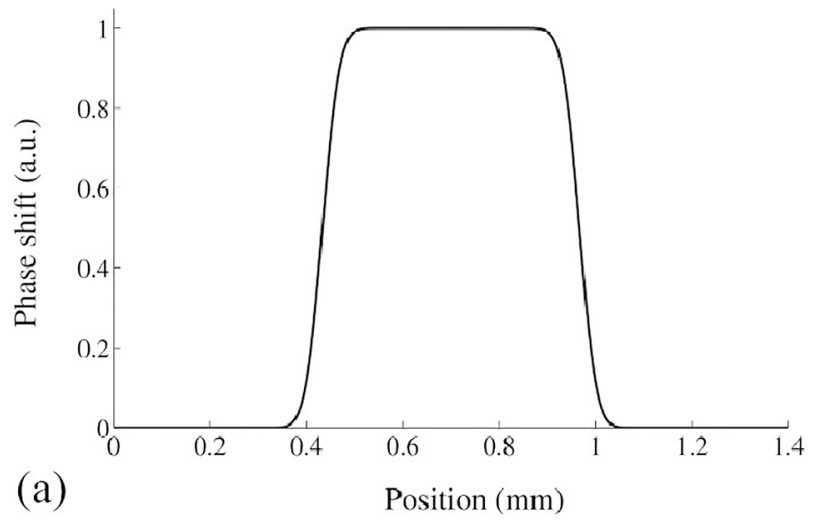

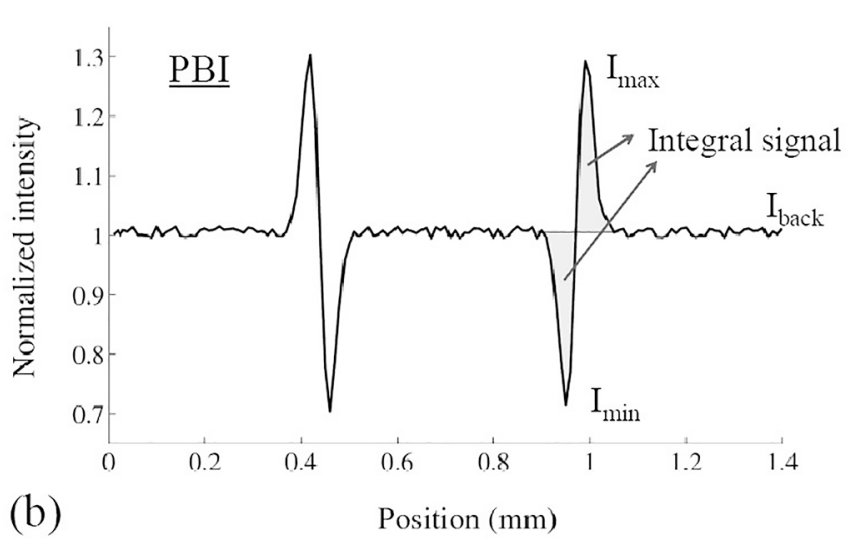

(a) transmitted beam. In $\mathrm{ABI}$, the analyzer crystal may be tuned to record the scattering signal or even to record the scattered and transmitted beams simultaneously. The generic term for these modalities is darkfield imaging (DFI) [19].

For the present discussion phase-contrast imaging is concentrated on propagation-based imaging (PBI), as it is the simplest method because no X-ray optical devices are needed. The source must be small to provide transverse (spatial) coherence of the beam, but longitudinal (temporal) coherence is not critical, so that wide-band radiation from conventional sources can be used. Sufficient distance between the object and the detector is required to separate the positive and negative parts of the Laplacian, as shown in Fig. 1. There are several methods for phase retrieval from the observed intensity distribution, which also includes the effects of absorption. Phase is the additive factor which is needed for computed tomographic (CT) reconstruction of the object morphology. There are recent analyses and comparisons of the methods [20-22]. Compared to methods where the phase gradient is usually observed in one direction only, PBI has the advantage of $2 \mathrm{D}$ recording of the Laplacian. Due to its experimental simplicity, PBI is probably the method with the widest potential for clinical application. A typical setup is shown in Fig. 2. Some applications to lung imaging are discussed in Section 4.

\subsection{Phase-contrast speckle imaging}

The air/tissue interfaces in the lung produce strong phase gradients and image contrast. Coherent radiation creates a speckled intensity pattern at the detector as a result of interference effects. When there are many overlapping alveoli, which act as compound refractive lenses, the image is a pattern of bright and dark spots (speckles). The pattern can be interpreted in terms of geometrical optics, which carries information

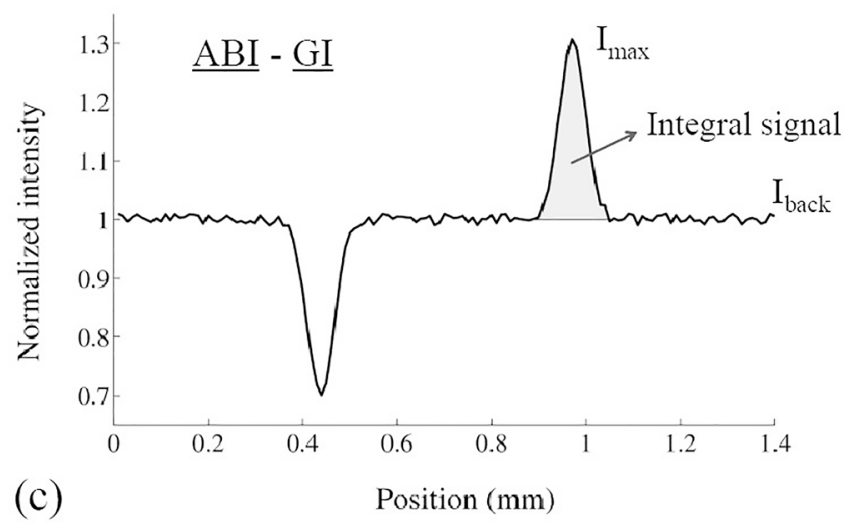

Fig. 1. (a) Profile of a pure phase object. (b) Typical corresponding propagation-based imaging (PBI) signal which is proportional to the second derivative of the phase; (c) Typical analyzer-based imaging (ABI) or grating-based imaging (GI) signal. Poissonian noise has been added. Reproduced from: [18] 


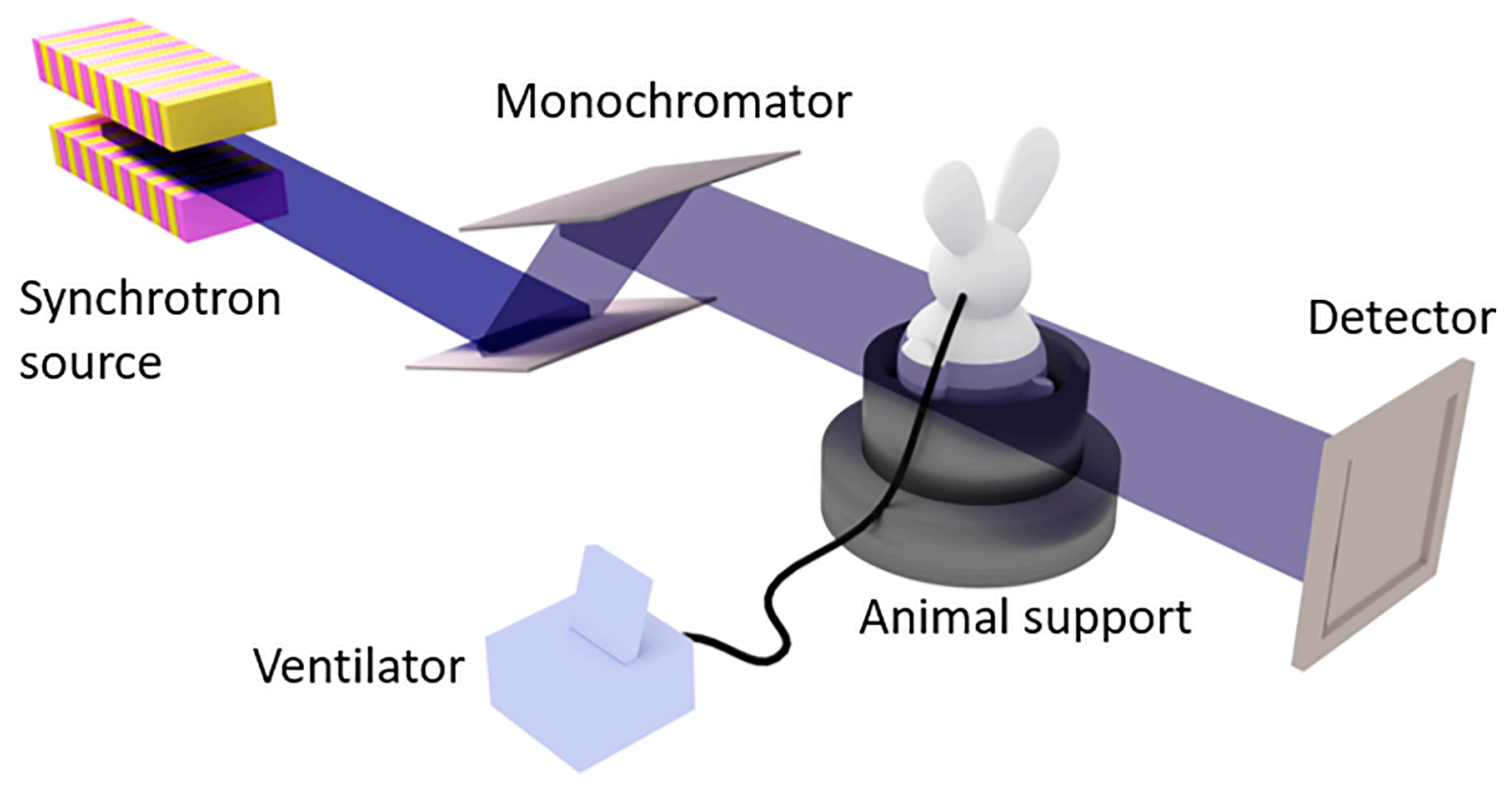

Fig. 2. Schematic view of the setup for Phase-contrast Imaging.

about the structure of the lungs [9]. It has been shown that under the plausible assumption that the alveoli are isolated spherical cavities the power spectrum of lung speckle in Fourier space is directly proportional to the size of the alveoli [23]. This has made it possible to measure the alveoli throughout the respiratory cycle. Studies of prematurely born rabbits with this technique have shown that immature alveoli were more likely to collapse at birth than in mature lungs, and that speckle analysis provides a sensitive measure of this maturity [24]. It should be noted that this imaging method is different than the so-called speckle-based imaging, discussed in Section 5.1, where the imaged sample distorts the random speckle pattern induced by a diffusor object.

\section{3. $4 D X$-ray imaging}

4D imaging is 3D imaging by CT reconstruction as a function of time. A dynamical process is described by tomograms taken at consecutive time points to create a 3D movie. This has been facilitated by recent developments in data acquisition protocols and in X-ray instrumentation. Temporal resolution may be artificially increased by gating, i.e. by imaging different time points within the phase of an essentially periodic process. Applied to recording dynamic microscopic features under living conditions, and in particular microscopic aspects of pulmonary inflation, several physiological aspects must be considered to optimize the data acquisition protocol.

The imaging protocol is based on the use of propagation-based phase-contrast imaging (PBI), where the tissue density gradients are emphasized to produce high contrast, which far exceeds the absorption contrast in conventional radiography. The radiation dose may be decreased in proportion, and a shorter exposure time is required to capture the motion of a microscopic feature of interest. A highly brilliant and coherent radiation source is needed, and in addition, a flexible imaging end-station is needed for precise triggering of all components of the image acquisition process. This is particularly critical in lung imaging. Breathing motion can be regulated by means of mechanical ventilation and deep anesthesia, but the irregularities of heartbeat alters the condition of strict motion periodicity which is necessary for standard gating techniques. Imaging the lung in-vivo with 3D resolution of alveoli and septa must ensure the precise capturing of the lung motion at identical heartbeat and respiratory phases. The problem may be solvable with grossly redundant tomographic data, but this might require X-ray doses that are not tolerated by the tissue, so that $4 \mathrm{D}$ imaging of lung at micrometer level may remain terminal experiments. The conditions and technical solutions have been discussed recently by several authors $[5,25,26]$.

\subsection{K-edge Subtraction imaging (KES)}

Conventional clinical X-ray imaging is based on absorption contrast which arises from variations in the elemental composition and density of the object. The elemental composition of soft tissue is rather uniform, and the absorption contrast is weak and largely due to variations in tissue density. New methods based on phase-contrast were discussed above, but contrast can also be enhanced by introducing a heavy element compound in the object.

Subtraction imaging is a generic term of X-ray radiography where an organ is visualized by the distribution of a contrast medium. Ideally, two images are acquired, the images being identical except in places where the beam or beams have traversed volumes containing the contrast medium. In 2D projection radiography, the difference image is the image of the contrast medium distribution. The difference may be temporal when the reference (mask) image is acquired before introduction of the contrast agent to the organ being imaged. Problems arise from motion of the lung tissue, which blurs the contrast agent image. These problems are avoided in K-edge subtraction (KES) imaging where two images are acquired simultaneously with X-ray beams of energies that bracket the K-absorption edge of the contrast agent. The contrast due to anatomic details of tissue and bone disappear if the difference in the X-ray beam energies is sufficiently small (Fig. 3) [13,27].

Several methods have been introduced for extracting suitable energy bands from the conventional sources, including selective filtering, crystal reflection and fluorescence radiation [28-31]. The limitations are due to insufficient intensity and a too large energy separation of the beams, which leads to incomplete suppression of bone absorption in the subtraction image. These limitations may be alleviated by use of new photon-counting detectors and new intense X-ray sources and compact synchrotron radiation sources.

KES imaging with X-rays from conventional sources remained on the prototype and proof-of-principle level until the introduction of synchrotron radiation in medical imaging. Narrow energy bands of high intensity could be separated from the continuous spectrum of wiggler radiation. For example, in the case of angiography imaging, $100 \mathrm{eV}$ wide beams are separated by $300 \mathrm{eV}$ and cross at the object. The attenuation factors can be written for both beams, and the mass densities of the tissue (plus bone) and the contrast agent are solved from the difference 


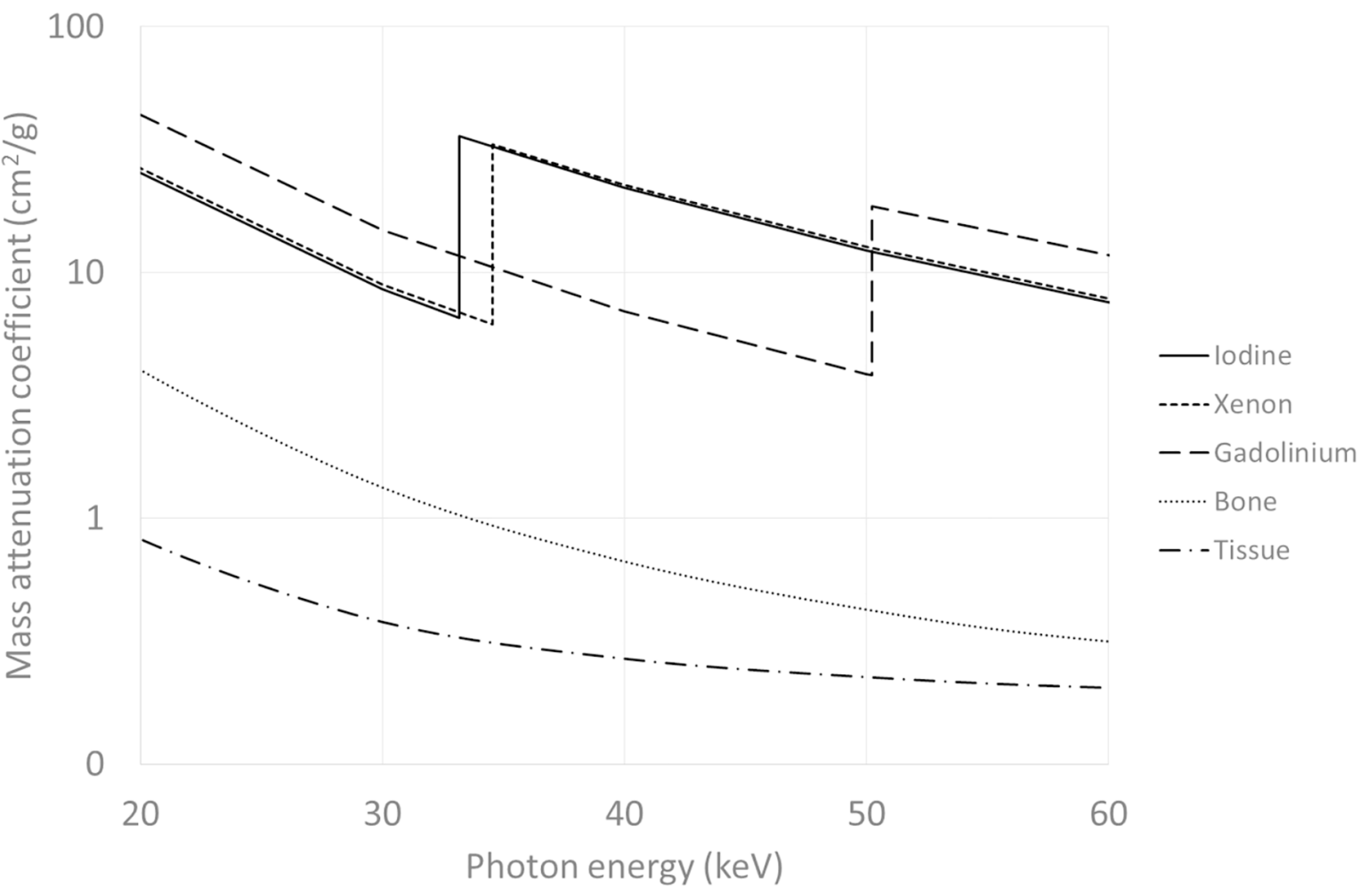

Fig. 3. Mass attenuation coefficients of iodine, xenon, gadolinium, bone and soft tissue [7]. Reproduced with permission from Elsevier.

image. In practice, minor corrections are needed to remove artifacts of strong contrast at bones and to eliminate the effects of multiple harmonic energies from the monochromator.

The current KES imaging set-ups are based on the use of a focusing perfect crystal monochromator, shown in Fig. 4. The cylindrically bent transmission (Laue-type) crystal acts as a lens and focuses in the vertical direction a band of radiation at the object. Two separate beams are obtained by placing a splitter in the middle of the beam. The bent crystal has another function. When a pencil beam travels through an asymmetrically cut crystal the reflection angle changes gradually, so that the reflectivity curve is broadened and the intensity is increased typically by an order of magnitude $[32,33]$. The transmitted beams are detected by a dual-line solid state detector, which has typically horizontal pixel sizes of a fraction of a mm. Three-dimensional images are obtained by standard CT methods from a large number of projection images when the object is rotated with respect to the X-ray beams. One important advantage of KES imaging is that the absolute concentration of the contrast material is obtained, as shown in Fig. 5. This allows for

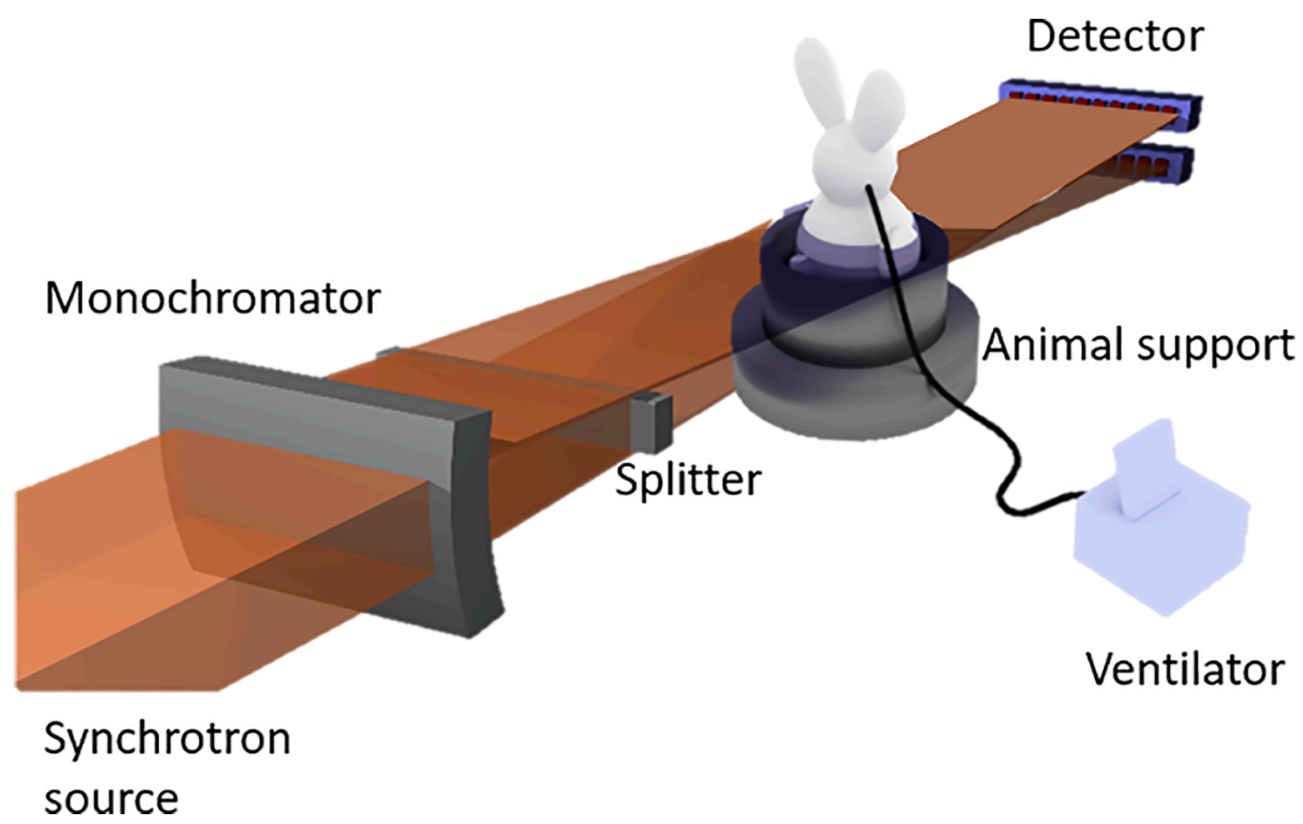

Fig. 4. Schematic view of the set-up for K-edge imaging. Two monochromatic beams cross at the object, and have energies that bracket the K-edge of the contrast agent. 

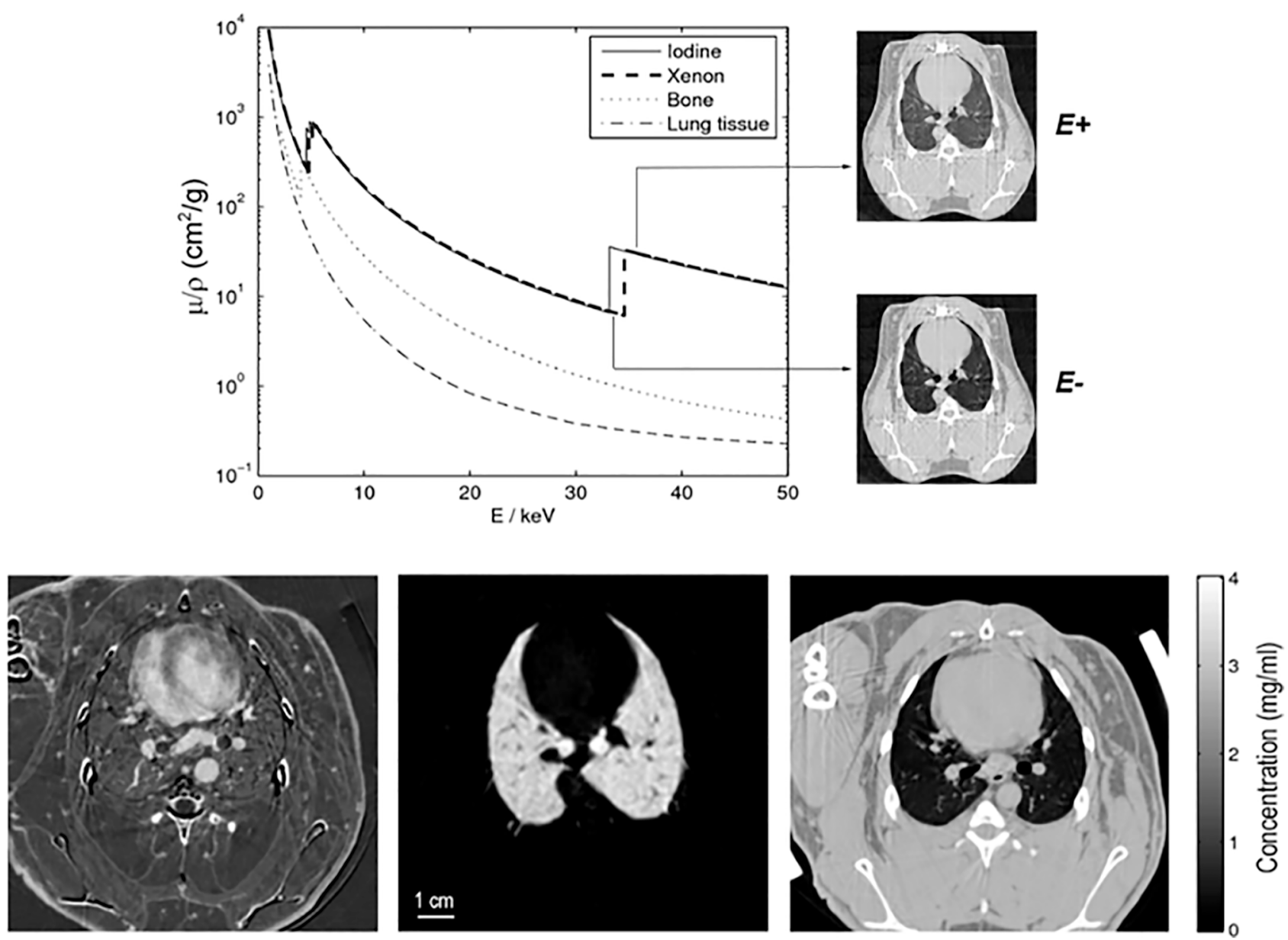

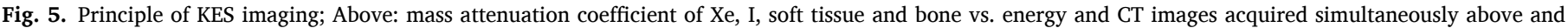
below the K-edge of Xe; Below: quantitative distribution images, respectively of I, Xe and tissue.

quantitative measurements of sensitivity and selectivity. Under typical imaging conditions of human brain imaging with iodine as the contrast agent the sensitivity is about $0.1 \mathrm{mg} / \mathrm{ml}$, which is about 10 times better than with filtered broad-band radiation $[34,35]$.

\subsection{KES functional lung imaging}

Imaging of regional pulmonary function is essential in studies of lung physiology and lung diseases. Traditional measurements of lung function yield parameters for the entire lung although it is known that ventilation distributions are inhomogeneous. Different imaging methods have been introduced to measure regional lung functional parameters such as ventilation, perfusion, inflammation and tissue biomechanics. These include magnetic resonance imaging of regional lung ventilation with hyper-polarized ${ }^{3} \mathrm{He}$ or ${ }^{129} \mathrm{Xe}$, or techniques based on inhalation or injection of radioactive tracers such as lung scintigraphy, single photon emission computed tomography (SPECT) and positron emission tomography (PET) [36-38]. However, X-ray CT remains the clinical method of reference for imaging the lung parenchyma, and quantitative analysis of lung CT images provides surrogate endpoints that are increasingly used in clinical trials to assess response to therapy [39]. Inhaled and injected contrast media allow detecting regional lung ventilation and perfusion $[36,40]$. Lung CT images acquired at more than one volume allow the assessment of regional lung deformation and strain [41]. Owing to its unique properties, structural and quantitative analysis of regional lung function can be performed at a much higher spatial and temporal resolution with synchrotron radiation than with clinical CT machines.
KES was introduced in lung imaging using stable Xe gas, originally as an alternative method of bronchography. The feasibility of Xe-KES in human imaging was first demonstrated by Giacomini and collaborators in 1998 [42]. Later, this approach was used to study different pulmonary diseases in animal models [25]. Xenon is the next element to iodine in the periodic table, so that the X-ray instrumentation initially developed for coronary angiography at the iodine K-edge was easily adapted to imaging at the Xe K-edge (34.56 keV). In vivo imaging with synchrotron radiation requires dedicated instrumentation and remote control. The synchrotron beam is a stationary horizontal fan so that the animal is subjected to rotation up to $180^{\circ} / \mathrm{s}$. Respiration is controlled by mechanical ventilation while the physiological parameters are continuously monitored and recorded.

Gas transport is driven by pressure gradients in the conducting airways and by diffusion, mainly in the acinar airways [43]. In volumecontrolled mechanical ventilation, a certain tidal volume of gas is inhaled and exhaled periodically. The alveoli are filled gradually by the marker gas [44]:

$D(t)=D_{a s}\left\{1-\exp \left[-\left(t-t_{0}\right) / \tau\right]\right\}$

where $D(t)$ is the gas density as function of time $t, D_{a s}$ the asymptotic density and $\tau$ the time constant. As ventilation within a lung region increases, the time constant of regional Xe washin or washout become shorter. Specific ventilation is defined as the initial gradient of the marker gas density divided by the asymptotic density:

$\left[(d D / \mathrm{d} t)_{0}\right] / D_{a s}=1 / \tau$.

Specific ventilation is ventilation per unit of regional gas volume, 
while the absolute ventilation can be obtained by the product of specific ventilation and the regional gas volume.

The spatial resolution and contrast sensitivity of KES imaging is largely determined by the characteristics of the detection system. For example, in many studies performed at the ID17 beamline of the ESRF, using a solid-state cooled germanium detector a pixel size of $0.33 \mathrm{~mm}^{2}$ and a sensitivity better than $0.1 \mathrm{mg} / \mathrm{ml}$ could be obtained. However, smaller pixel sizes of $47 \mu \mathrm{m}^{2}$ have previously been achieved in in vivo KES-CT imaging using a charge coupled device (CCD) detector [45]. This makes KES a unique method because of the high spatial resolution and absolute scale of the ventilation distributions.

\subsection{Extensions of KES imaging}

Understanding the lung function on the alveolar level requires highresolution mapping of ventilation and perfusion simultaneously. This would be possible in KES imaging by using two contrast agent imaging, i. e. iodine for perfusion and xenon for ventilation. New instrumentation is being developed so that both absorption edges could be reached at the same time. In spectral imaging a wide band of radiation is focused on the organ being imaged, and the energies are separated by an energy discriminating detector or by dispersing the spectrum on the detector in such a way that for an elemental X-ray there is a one-to-one correspondence between energy and position on the detector $[46,47]$. The energy range depends on the focal lengths of the monochromator and the divergences of the X-ray beam. In principle, the method can be used with any source of continuum radiation, but so far the applications have been based on use of synchrotron radiation due to its high relative flux which reduces the necessary acquisition time. The small vertical divergence of the beam limits the spectral range typically to less than $1 \mathrm{keV}$, but focusing the radiation fan in the horizontal direction provides a band of radiation that can cover the K-edges of iodine and xenon. A disadvantage of spectral imaging is the large radiation dose when the energy band of the focused beam is large. For the actual imaging of the contrast agent distributions, only narrow bands in the vicinities of the K-edges are of interest. In the case of simultaneous imaging of iodine and xenon only two pairs of about $0.1 \mathrm{keV}$ wide and separated by $1.5 \mathrm{keV}$ are needed. The unused part of the beam could be blocked by a selective beam stop.

Another method of simultaneous imaging of ventilation and perfusion with iodine and xenon as the respective contrast agents is a combination of KES and temporal subtraction imaging (TSI). In this method KES imaging of ventilation is performed by a dual-energy beam that brackets the xenon K-edge but where both energies are above the iodine K-edge [48]. The concentration of xenon is solved, effectively subtracting the contribution of iodine and tissue. With the xenon concentration known, its contribution to the attenuation coefficient image can be subtracted. The remaining images are used in temporal subtraction to yield the iodine concentration. In such an experiment the iodine contrast agent is continuously infused to achieve a steady state, and the cyclic changes in lung perfusion that occur as a result of cardiac pumping are not resolved. Using this method, the imaging sequence could yield timeaveraged regional distributions of pulmonary blood volume and perfusion as well as regional ventilation. The mismatch of these two processes is one of the most frequent causes of abnormal gas exchange in lung disease.

\subsection{Compact Compton sources}

Over the past 2 decades, Compact Compton Sources (CCS) have been developed which offer several new possibilities for imaging, including potential use in clinical environments. A CCS is based on a process where a high-energy electron beam collides with a laser beam which is scattered back as short wavelength X-rays [49]. Electron bunches are produced in a linac or accelerated in a small storage ring, and the laser pulse is amplified in a Fabry-Perot resonator. The electrons and laser photons collide in the interaction region, producing a narrow cone of X-rays. The process can be described as a collision between two particles, or inverse Compton scattering. The X-ray beam has a Lorentzian energy distribution, which is suitable to many imaging applications. In a typical case the energy of the infrared laser photon is $1.2 \mathrm{eV}$, the electron energy 44 $\mathrm{MeV}$, and the maximum X-ray energy is $35 \mathrm{keV}$. The X-ray energy is tunable to the K-edge energies of iodine and xenon by changing the electron energy. At half-energy the radiation cone half-opening angle is $12 \mathrm{mrad}$. Due to the actual directional and spatial distributions of the colliding beam the energy distribution is rather uniform on the axis of the radiation cone. In a typical case the relative bandwidth is $>2 \%$ in a radiation cone of 2 mrad opening. In standard KES two narrow energy bands could be produced by a bent Laue-type monochromator.

There is one commercially available CCS machine [50], which has been utilized in phase-contrast imaging [51-53] and KES [54]. Several construction projects are underway [49] with target X-ray fluxes from $10^{12} \mathrm{ph} / \mathrm{s}$ to $10^{14} \mathrm{ph} / \mathrm{s}$. These fluxes are comparable with the fluxes of bending magnets or even wiggler sources of synchrotron radiation facilities. It has been estimated that a CCS operating at high energies can also be used for radiation therapy [55].

\section{Applications}

In the following, examples of synchrotron radiation imaging of lung function using the two main techniques, namely phase-contrast imaging (PCI) and K-edge subtraction imaging, will be discussed.

\subsection{Phase-contrast imaging applications}

The lung poorly attenuates $\mathrm{x}$-rays. However, the numerous air-tissue interfaces within the lung airways and alveoli result in refraction and phase changes of the incident X-rays. Phase-contrast $\mathrm{x}$-ray imaging (PCI), also referred to as 'refraction-enhanced' imaging [56] uses the phase information in addition to attenuation to enhance contrast within poorly-attenuating structures. In addition to improving contrast, this imaging approach has the advantage of reducing the radiation dose in comparison to conventional x-ray attenuation imaging [10]. The numerous air-tissue interfaces crossed by the incident beam within the lung produce phase gradient patterns which resemble random noise or "speckles", unlike the surrounding soft tissues. Previously, highresolution 3D synchrotron phase-contrast x-ray imaging $\left(2.8 \mu \mathrm{m}^{3}\right.$ voxel size) has been used in post-mortem mouse to track acinar deformation during lung inflation [57]. This method has shown the differential expansion of alveolar ducts and alveoli depending on airway pressure, with alveolar ducts expanding predominantly at pressures below $\sim 8 \mathrm{cmH}_{2} \mathrm{O}$. This approach has been combined with computational fluid dynamics modelling of aerosol particle deposition $[58,59]$.

In 2D-projection imaging, overlapping alveoli act as aberrated compound refractive lenses [9] resulting in the characteristic speckle pattern which is closely linked to the underlying tissue architecture. By placing a small animal in a tube of water during imaging, it is possible to accurately measure changes in regional lung air volume [60]. The relationship between the peak area and the lung volume is not simple, but it has been shown that absolute regional lung air volume can be calculated by first calibrating the speckle against known lung air volumes [60]. The technique has an excellent time resolution; however, it is limited by the fact that only $2 \mathrm{D}$ projection images can be acquired. Therefore, the structure of individual acini cannot be studied directly. The techniques for quantifying lung structure and function from the phase-contrast speckle have been used to quantify real-time changes of lung liquid clearance and aeration at birth in rabbits. These studies have been crucial for understanding the transition of the cardiopulmonary system to life after birth. The findings have guided development of safer methods of resuscitating premature infants at birth who fail to breathe without medical intervention [25].

More recently, Broche et al. [61] have used PCI to investigate cyclic 
alveolar recruitment/derecruitment phenomena during breathing, using sequential PCI at subacinar resolution $\left(47.5 \mu \mathrm{m}^{3}\right.$ voxel size $)$ over short time intervals of $\sim 1.5 \mathrm{~min}$, at end-expiratory pressures ranging from 0 to $12 \mathrm{cmH}_{2} \mathrm{O}$ in a model of acute respiratory distress syndrome (ARDS) in anesthetized rabbits. This pathologic condition is characterized by a heterogeneous increased-permeability edema due to acute inflammation. This condition causes severe gas exchange alterations and increased lung tissue stiffness which increases the work of breathing. Patients with ARDS often require mechanical ventilation in order to maintain adequate gas exchange and to reduce the work of breathing. However, positive pressure ventilation imposes mechanical stresses on the parenchyma that can worsen lung injury, a condition known as Ventilator-Induced Lung Injury (VILI). The exact mechanisms leading to VILI at the microscopic level remain elusive, although it is generally accepted that positive-pressure mechanical ventilation imposes exaggerated mechanical stress on the lung tissue, which can in turn worsen inflammation and lung injury. Cyclic reopening of lung regions that become derecruited upon expiration, a phenomenon thought to induce "atelectrauma", can also contribute to the expansion of lung injury due to high stresses normal to the epithelial surface of the airways as they reopen. High stress is also produced at the interface between adjacent collapsed and aerated alveoli, leading to increased local stress or 'stress concentration'. The study of lung micromechanics under assisted ventilation requires volumetric images of the lung structure at high resolution.

The extent and spatial distribution of recruitment/derecruitment was analyzed by registering and subtracting subsequent volumetric images (Fig. 6). The authors found alternating patterns of recruitment/ derecruitment occurring within the same time frame in adjacent and communicating airspaces subtended by the same terminal airway. They combined their data with computational modelling of recruitment/ derecruitment phenomena in a realistic rabbit lung model based on the segmentation of a full bronchial tree, where opening and closure of individual airways were determined not only by a critical pressure, but also occurred with a characteristic speed rather than instantaneously [61].

Propagation-based phase-contrast computed tomography with a $47.5 \mu \mathrm{m}^{3}$ voxel size has been used at various positive end-expiratory pressures in a model of ARDS in anesthetized rabbits. Cross-sections of the same individual airways were measured at the different pressures (Fig. 7) [62]. Airway collapsibility increased in the injured lung with a significantly faster drop in airway cross-section as airway pressure decreased in small airways ranging between 210 and $1690 \mu \mathrm{m}$ in radius at $12 \mathrm{cmH}_{2} \mathrm{O}$.

The main mechanism of airway closure in initially patent airways in ARDS was identified as "compliant collapse". An example is shown in Fig. 7. Theoretical studies suggest that this mechanism involves an instability in the thickness of the fluid lining layer of the airway wall. Where this fluid layer thickens, the compressive forces that are generated cause a buckling of the airway wall that can lead to the collapse of the airway lumen along a certain length. Moreover, the analysis of airway cross-sections vs. length revealed that closure can occur at more than one site, sometimes leading to gas trapping and distension of the lumen. Evidence of compliant collapse of airways in ARDS is indicated by the involvement of fluid layer movements at the microscopic level. This suggests that airway closure and reopening is not merely dependent on critical opening and closing pressures but is also a highly dynamic, time-dependent phenomenon [62]. The above findings have
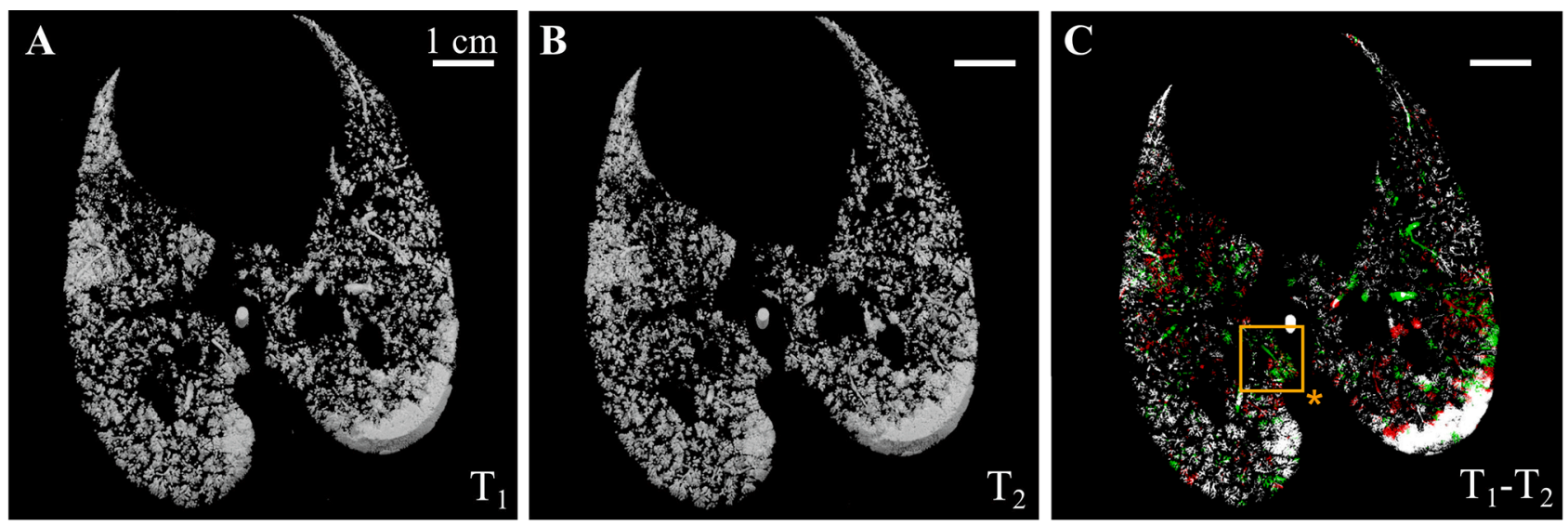

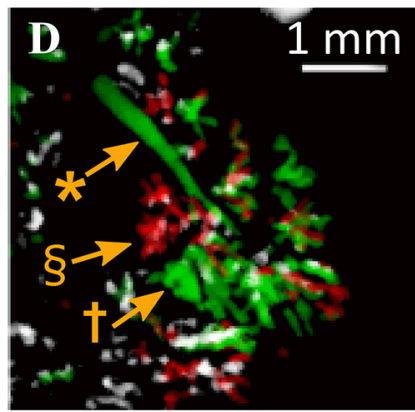

$\mathrm{T} 1-\mathrm{T} 2$

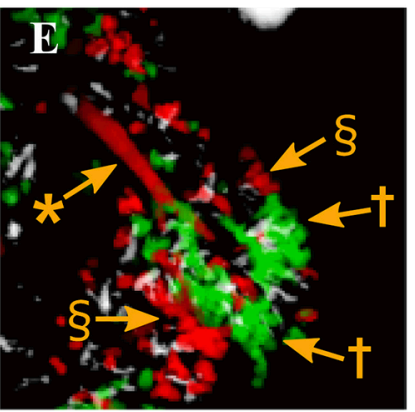

T2-T3

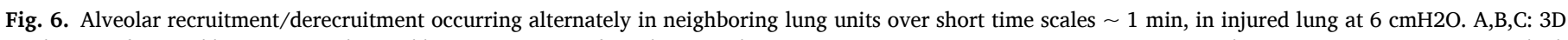

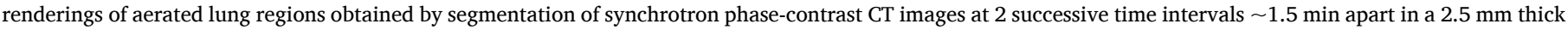

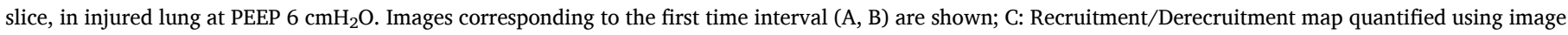

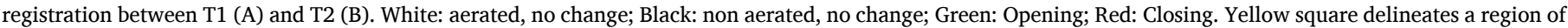

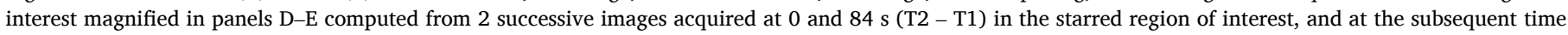

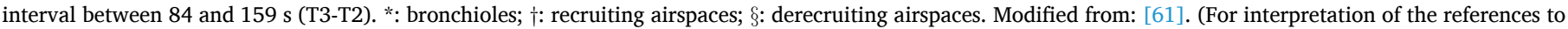
color in this figure legend, the reader is referred to the web version of this article.) 

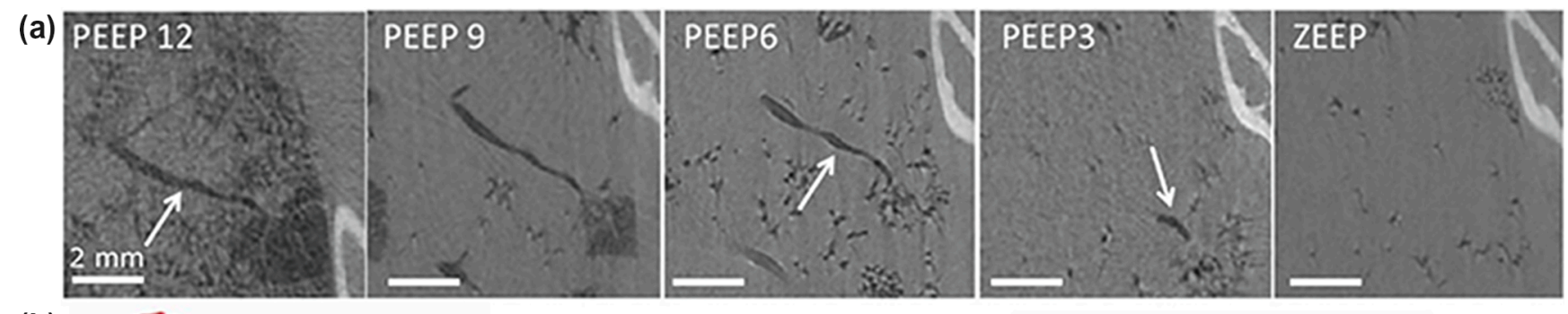

(b)
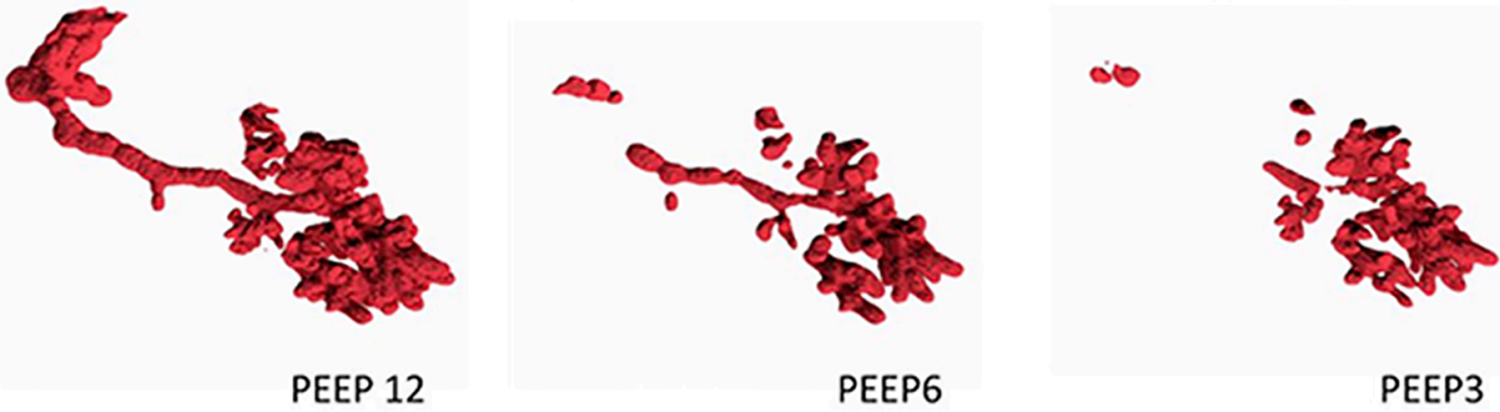

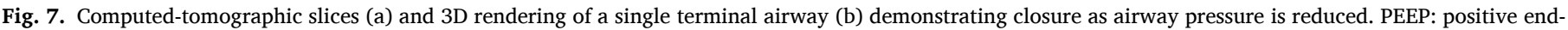
expiratory pressure, ZEEP: zero end-expiratory pressure in an in vivo model of ARDS. Modified from: [62].

implications for patients with ARDS under mechanical ventilation because they suggest that strategies aiming at reducing the time during which respiratory pressure is lowered during the breathing cycle may help prevent airway closure.

Studies $[63,64]$ show that insight into the lung micromechanics of the normal and injured lung can be gained using in vivo phase-contrast synchrotron radiation tomography since the technique allows the measurement of regional alveolar aeration with high contrast sensitivity, short acquisition times compatible with in vivo imaging, and the ability to acquire 3D data at subacinar spatial resolution. However, the spatial resolution of static imaging approaches is limited by motion blurring due to cardiac and vascular motion, which hampers the study of aeration and deformation within individual alveoli.

This issue is addressed by 4DCT imaging. This approach involves gating the acquisition of individual image projections with the cardiac and respiratory motions. Moreover, the exposure time of each image projection needs to be reduced as the microscopic features of interest become smaller, which makes 4DCT microscopy technically challenging [25]. The high photon flux and coherence of synchrotron radiation are necessary for this technique, as well as accurate physiologic signal acquisition and control software allowing precise triggering of all components of the image acquisition process [65]. Resolving alveolar structure in the lung is particularly challenging due to inhomogeneous speed and magnitude of the physiological tissue motion. Acquiring abundant tomographic data can help resolve this issue.

Under static lung inflation conditions, Lovric et al. studied lung inflation patterns during diastole at the alveolar scale in vivo, with a voxel size of $2.9 \mu \mathrm{m}^{3}$, in anesthetized 9-day old rats. They acquired 450 individual projections at $3 \mathrm{~ms}$ exposure time, for a total acquisition time of $2 \mathrm{~min}$. Their data demonstrate the feasibility of eliminating motion artefacts due to cardiac activity and resolving alveolar structure in vivo [5].

More recently, Fardin et al. investigated lung tissue deformation induced by cardiac contractions in anesthetized rabbits at $20 \mu \mathrm{m}^{3}$, showing the regional inhomogeneity in heart-induced lung deformation [66]. Using this methodology both the respiratory and cardiac-induced motion could be resolved at $3 \mu \mathrm{m}^{3}$ voxel size in anesthetized rats [67]. Also, 4D tomographic microscopy at $2.9 \mu \mathrm{m}^{3}$ pixel size has been used to study the progression of ventilator-induced lung injury in anesthetized $\mathrm{Balb} / \mathrm{c}$ mice [68].

These studies have shown that $4 \mathrm{D}$ tomographic microscopy is a valuable technique for studying regional lung structure and function at microscopic length scales. The main limitations of the technique are the length of data acquisition ranging up to tens of minutes, and the risk of excessive radiation dose which can alter the underlying tissue structures. More sensitive detection devices and specifically designed imaging end stations can help mitigate these limitations in the future.

\subsection{K-edge subtraction imaging applications}

K-edge subtraction imaging provides quantitative maps of a contrast element while simultaneously providing images of the underlying tissue structure. Using this approach, inhaled Xe gas distribution can be measured within the pulmonary alveoli [12], while the injection of Iodinated contrast elements allows the quantification of the regional blood volume distribution and perfusion [48].

By imaging the washin or washout of Xe gas during sequential breaths, the regional distribution of specific ventilation can be imaged and quantified as specific or absolute regional ventilation. The distribution of regional ventilation is inhomogeneous in normal lungs and this inhomogeneity increases dramatically under pathologic conditions. The uneven distribution of ventilation has major fundamental and clinical implications. Ventilation heterogeneity affects the matching of regional ventilation and perfusion leading to less efficient gas exchange, and can significantly affect the apparent degree of mechanical obstruction. Moreover, ventilation distribution importantly determines the distribution of inhaled medications and inhaled environmental pollutants. One of the lung diseases where ventilation distribution inhomogeneity is enhanced is asthma. Asthma is characterized by the exaggerated contractile response of airways to various stimuli. For asthma diagnosis, airway responsiveness is often investigated using pharmacological substances that directly stimulate the airway smooth muscle. In asthmatic individuals however, airways constriction is indirectly induced by stimulation of inflammatory cells such as mast cells and eosinophils that release constricting mediators. Using KES imaging, it was demonstrated that when airway constriction is induced by the inhalation of histamine mimicking an asthma attack in anesthetized rabbits, regional ventilation becomes very heterogeneous with development of patchy areas of reduced ventilation $[44,69,70]$. It was shown that the kinetics of airway response to histamine was very different in central versus small peripheral airways, with faster constriction and recovery in the lung periphery. Central airways were slower to reach their maximal constriction 
[44], occasionally showing paradoxical dilatations [71]. This phenomenon may be due to the dynamic tidal expansion of proximal bronchi when distal airways constrict downstream which has been termed serial airway interdependence [72].

The mechanism through which airway smooth muscle constriction is induced results in radically different patterns of airways constriction [69]. In rabbits sensitized to ovalbumin, airway constriction was induced either non-specifically through a pharmacological agent such as methacholine, which acts directly on the airway smooth muscle, or specifically through an allergen such as ovalbumin following allergic sensitization, which constricts the airways indirectly by triggering the activation of mast cells and eosinophils. While methacholine induced mainly central airway constriction, the allergen caused a much more heterogeneous pattern of ventilation distribution in the lung periphery through constriction of peripheral airways (Fig. 8). In that study, respiratory impedance was measured using the forced oscillation technique. A model that includes resistance, tissue damping and elastance was fitted to the impedance data [73]. Responses in airway resistance and tissue elastance measured by the forced oscillation technique were demonstrated to be related to the central and peripheral airway responses, respectively [69]. Since both constricting agents were injected in the blood, the more inhomogeneous airway reaction in allergic animals may have been due to the local heterogeneity of the distribution of the immune cells involved in the allergic reaction itself. This was investigated using KES imaging in ovalbumin-sensitized Brown-Norway rats, a species genetically prone to develop allergic sensitization [45]. When the animals were exposed to allergen, and subsequently imaged while breathing a Xe and $\mathrm{O}_{2}$ gas mixture, patchy regions of ventilation defect developed in the lung periphery. Histologic examination of matching slices of the lung tissue revealed that total cellular, eosinophil and mast cell infiltrations were stronger within compared to outside the lung regions with reduced ventilation. Moreover, the inhomogeneous distribution of an inhaled aerosol containing environmental antigens or constricting agonists can result in an inhomogeneous peripheral airway response. More recently, the regional deposition of aerosol particles containing iodine was quantitatively mapped in rabbit lungs [74]. Ventilation distribution using KES imaging during Xe washin, and the lung airway branching morphology, both critical parameters in determining aerosol transport and deposition, were also imaged. This study showed that the deposition of aerosol particles of a median aerodynamic diameter of $\sim 3 \mu \mathrm{m}$ was strongly heterogeneous (Fig. 9), decreased following methacholine-induced airway constriction, and that deposition distribution could be imaged repeatedly with KES to obtain data on the kinetics of regional deposition with time, or to determine how interventions can change the deposition pattern of aerosol particles.

Overall measurements of lung function do not allow assessing the functional behavior of the lung periphery under mechanical ventilation. Here, KES imaging can be used to investigate how changing mechanical ventilation strategies such as alveolar recruitment and positive endexpiratory pressure (PEEP) affect this behavior. Using this technique, PEEP was shown to improve aeration in collapsed lung regions but not to eliminate overventilation of normal lung units in a model of experimental lung injury in rabbit [75]. Combined measurements of lung aeration based on tissue-density images, and regional ventilation based on Xe washin, allows distinguishing regions of alveolar collapse that are tidally ventilated, exposing them to concentration of mechanical stresses [76], or regions with full or partial gas trapping, while static CT images do not allow this distinction [75]. Moreover, the administration of exogenous surfactant [77] or the impact of different modes of mechanical ventilation such as volume control or pressure-regulated modes on regional lung function, can be assessed by KES imaging [78].

Mechanical ventilation also affects regional lung function in normal lung, for example in subjects undergoing general anesthesia for surgery. Particularly, the regional distribution of blood volume in the lung can be tidally modified due to the increased pressure upon inspiration. Despite the importance of dynamic changes in the regional distributions of gas and blood during the breathing cycle for lung function in the mechanically ventilated patient, no quantitative data on such cyclic changes and their determinants were available. Using KES imaging, the cyclic variations in regional pulmonary blood volume during the respiratory cycle were demonstrated for the first time (Fig. 10), and dynamically imaged as a video animation [79]. The magnitude of the cyclic changes in regional blood volume was significantly affected by gravity, and by mechanical ventilation settings such as the level of PEEP. The significance of these phenomena is their potential impact on the dynamics of tidal gas exchange, which can be further investigated by KES imaging.

\section{Clinical translation}

\subsection{X-ray phase-contrast imaging}

Although PCI is applicable in human subjects [80], no functional
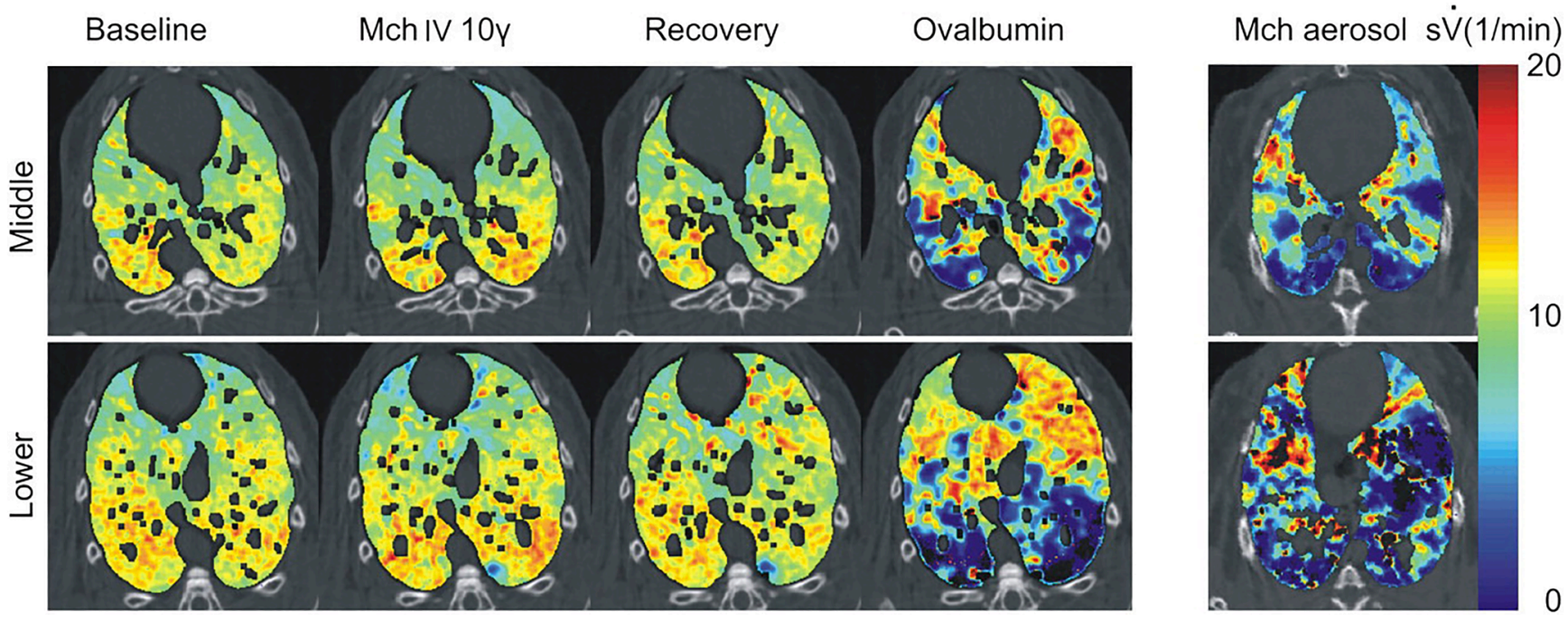

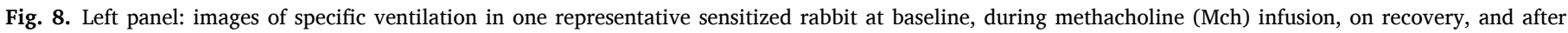

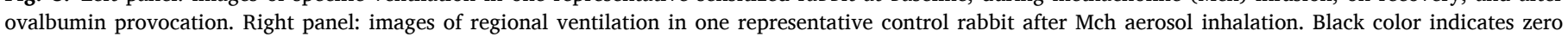
specific ventilation. .

Reproduced with permission from: [69] 

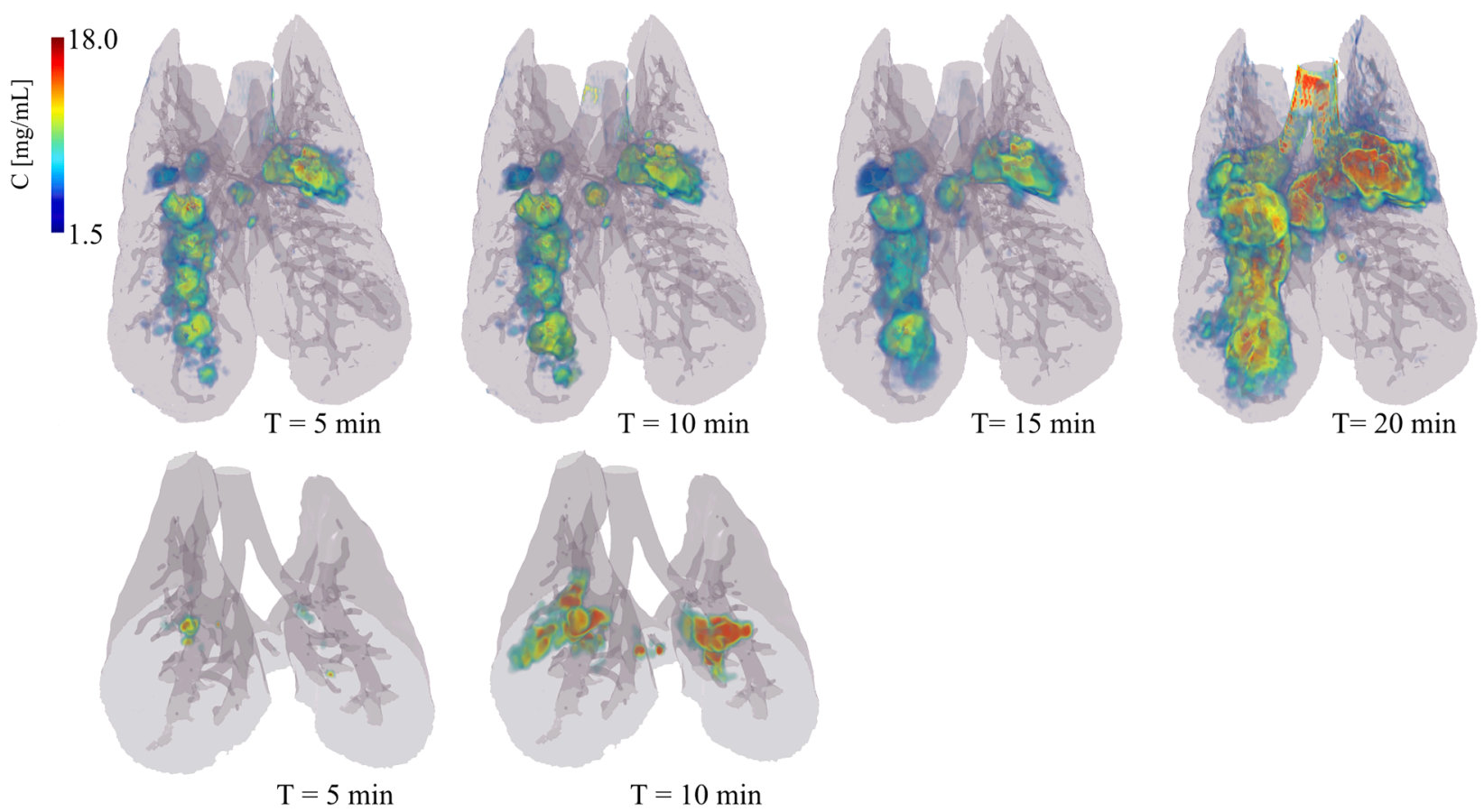

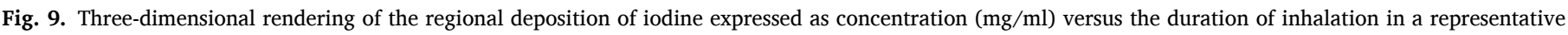

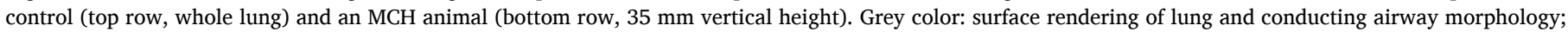

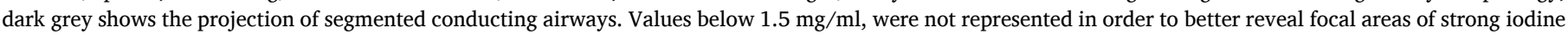

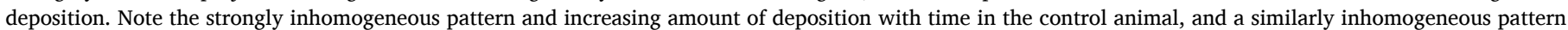
but less overall deposition in the MCH animal. .

Reproduced with permission from: [74]
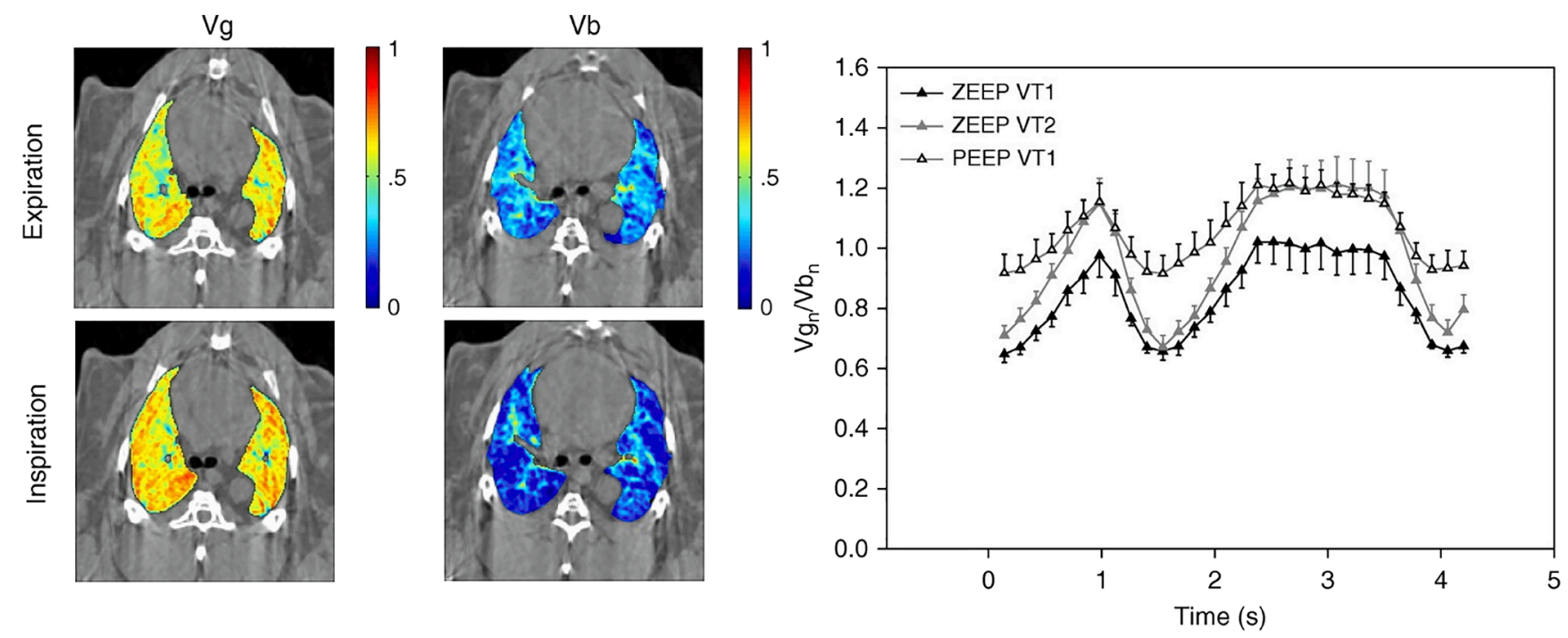

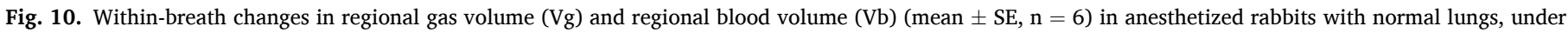

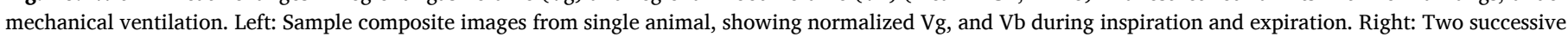

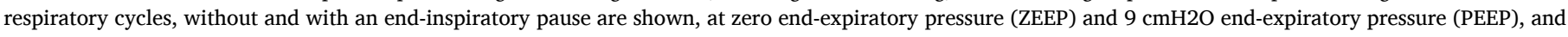
at 2 different tidal volume levels (VT1 and VT2). Modified from: [79].

lung imaging data in humans have been acquired to date. Because contrast enhancement due to $\mathrm{x}$-ray refraction far exceeds that of attenuation particularly at higher energies, the radiation dose is not expected to be a major limiting factor. For example, PCI-CT performed at an entrance dose of $1.6 \mathrm{mGy}$ in ex-vivo human breast samples was found to have a better image quality than cone-beam CT with a standard source at $5.8 \mathrm{mGy}$ [81]. In translational functional lung CT studies in animals with optimized image quality rather than radiation dose, the latter is typically on the order of $160 \mathrm{mGy}$ per 1000 projections using a free propagation setup and a monochromatic beam at $50 \mathrm{keV}$ (unpublished data). It is the scarcity and cost of large synchrotron facilities that mainly hampers the utilization of synchrotron sources for functional lung imaging as a diagnostic tool. Over the past decades, Compact Compton Sources (CCS) have been developed, with one commercially available device [50] and several ongoing projects [49]. Such compact radiation sources may allow a wider availability of PCI for application in clinical investigation 
including for functional lung imaging. A recent study of dark field imaging (DFI) of the lung using a CCS in live pigs has suggested that this approach holds promise for human application with clinically acceptable parameters [82]. Alternatively, speckle-based imaging may make possible the use of a high-brightness liquid-metal-jet source to simultaneously obtain the spatial distribution of both the refractive index and absorption within a sample, making this imaging method available for widespread use.

The principle of speckle-based imaging is to quantify the effect of the sample on the speckle pattern induced by a random diffusor such as a piece of sandpaper, through a windowed correlation between a pair of images taken with and without sample $[83,84]$. This modality is not the same as the phase-contrast speckle imaging discussed in Section 3.2 above. Here, images of the near-field speckle pattern produced by a random diffusor, are obtained with and without the sample placed between the diffusor and the detector. The distortion of the near-field speckle pattern of the diffusor by the sample, allows retrieving phase and absorption images of the sample using a cross-correlation algorithm $[83,84]$. This method has been applied to static paraffin-embedded human lung samples [84] but so far not to functional lung imaging.

\subsection{K-edge subtraction imaging}

The possibilities and limitations of KES with synchrotron radiation have been extensively reviewed by Thomlinson et al. [13]. The feasibility of Xe-KES in human imaging was first demonstrated by Giacomini and collaborators in 1998 [42]. A study by Strengell et al. [85] has shown that within limitations given by the amount of Xe gas that a patient can tolerate given its sedative properties, as well as by the clinical radiation dose limitation for high-resolution chest CT, it should be possible to carry out human studies and potential human applications. The practical limitations in utilizing synchrotron sources for clinical studies with the KES method have so far limited functional lung imaging applications to pre-clinical animal studies. An alternative to monochromatic radiation is the use of clinical x-ray sources for dualenergy computed tomography (DECT). Although clinically available $\mathrm{x}$ ray tubes produce a poly-energetic spectrum, the general principle of KES remains valid. Algorithms have been developed to quantify the material composition of the imaged tissues and to differentiate contrast elements from the intrinsic composition. Various techniques and hardware have been developed for clinical scanners including rapid switching of the tube potential, dual x-ray sources, multilayer detectors or photon-counting detectors [86]. This approach has been used for functional imaging of regional lung ventilation using inhaled Xe in subjects with COPD, asthma and bronchiolitis obliterans [87]. However, radiation dose, overlap of energy spectra, noise, persistent beam hardening and the requirement for careful system calibrations limit its clinical use [86]. Currently, CCS are being investigated for KES imaging and a proof-of principle ex vivo study has shown the possibility to acquire KES images at iodine K-edge [54]. With the improvement in the intensity of radiation produced by CCS and improvements in detection systems, clinical application of KES using monochromatic radiation can be regarded as a realistic option in the future.

\section{Future directions and challenges}

Elucidating the 3D structure and real-time lung function at microscopic length scales in vivo is one of the most challenging applications of synchrotron radiation in biomedical imaging. Current data suggest that dynamic measurements allowing the study of regional lung structure and function using methods such as phase-contrast imaging or K-edge subtraction imaging are unique tools for better understanding of pathologic mechanisms, and for devising strategies to mitigate them. Synchrotron radiation imaging allows the investigation of lung micromechanics, regional gas distribution and ventilation, regional blood distribution and perfusion, for measuring gas and nanoparticle transport and exchange, aerosol deposition, nanoparticle toxicology and theranostics (diagnostic and therapeutic use), among other applications. The intensity and coherence of synchrotron beams allows phase-contrast imaging of weakly absorbing lung tissues and allows visualizing structural details at $\sim 1 \mu \mathrm{m}$ length scale which is inaccessible through traditional x-ray imaging techniques, while limiting the radiation dose. On the other hand, the ability to tune the energy of monochromatic $\mathrm{x}$ rays over a wide range allows K-edge subtraction imaging. This imaging modality allows direct quantification of the distributions of contrast elements that can be used as tracers. Real-time imaging of lung function is highly challenging, particularly in vivo due to motion blurring and the non-linear deformation of the lung tissue with breathing and cardiovascular motion. Other challenges include imaging large fields of view, while maintaining a degree of spatial resolution sufficient to resolve the structures of interest. There is currently a trade-off between spatial and temporal resolutions, and both are difficult to achieve simultaneously. In vivo synchrotron radiation imaging also faces limitations due to radiation dose, and a limited field of view for imaging large samples at most facilities. However, possibilities to achieve these challenging goals exist at current synchrotron facilities with recent progress in detection, acquisition and data processing capabilities allowing for quasi real-time 3D "tomoscopy" [88]. Although this technique is not directly applicable in vivo, fast detection, data transmission and real-time image processing capabilites are crucial for in vivo dynamic lung imaging.

A challenge posed by fast acquisition 3D imaging is handling the large volume of data, which can rapidly represent several terabytes. Large data volumes cannot be visualized in real time with conventional approaches. A change in the data representation paradigm, from the classical Cartesian grid to a hierarchical data structure is therefore mandatory to allow a real-time visualization on different planes as well as morphological analysis in a reasonable time. This in turn, requires adapting image processing algorithms.

\section{Acknowledgements}

The authors wish to thank Luca Fardin for his help with dose calculation.

\section{Funding}

This work was supported by University of Helsinki and the Tampere Tuberculosis Foundation, (LP, PS, WT), and by INSERM (SB) and the French National Research Agency (SB) in the framework of the "Investissements d'avenir" program (ANR-15-IDEX-02).

\section{References}

[1] Weibel ER. Principles and Methods of Morphometry. Morphometry Hum Lung 1963:9-39. https://doi.org/10.1016/b978-1-4832-0076-7.50008-9.

[2] Majumdar A, Alencar AM, Buldyrev SV, Hantos Z, Lutchen KR, Stanley HE, et al. Relating Airway Diameter Distributions to Regular Branching Asymmetry in the Lung. Phys Rev Lett 2005;95. https://doi.org/10.1103/physrevlett.95.168101.

[3] Glenny RW, Robertson HT, Yamashiro S, Bassingthwaighte JB. Applications of fractal analysis to physiology. J Appl Physiol 1991;70:2351-67. https://doi.org/ 10.1152/jappl.1991.70.6.2351.

[4] Lovric G, Barré SF, Schittny JC, Roth-Kleiner M, Stampanoni M, Mokso R. Dose optimization approach to fast X-ray microtomography of the lung alveoli. J Appl Crystallogr 2013;46:856-60. https://doi.org/10.1107/s0021889813005591.

[5] Lovric G, Mokso R, Arcadu F, Vogiatzis Oikonomidis I, Schittny JC, Roth-Kleiner M, et al. Tomographic in vivo microscopy for the study of lung physiology at the alveolar level. Sci Rep 2017;7. https://doi.org/10.1038/s41598-017-12886-3.

[6] Cloetens P, Barrett R, Baruchel J, Guigay J-P, Schlenker M. Phase objects in synchrotron radiation hard x-ray imaging. J Phys D Appl Phys 1996;29:133-46. https://doi.org/10.1088/0022-3727/29/1/023.

[7] Bravin A, Coan P, Suortti P. X-ray phase-contrast imaging: from pre-clinical applications towards clinics. Phys Med Biol 2012;58:R1-35. https://doi.org/ 10.1088/0031-9155/58/1/r1.

[8] Kitchen MJ, Paganin D, Lewis RA, Yagi N, Uesugi K, Mudie ST. On the origin of speckle in x-ray phase contrast images of lung tissue. Phys Med Biol 2004;49: 4335-48. https://doi.org/10.1088/0031-9155/49/18/010. 
[9] Kitchen MJ, Lewis RA, Yagi N, Uesugi K, Paganin D, Hooper SB, et al. Phase contrast X-ray imaging of mice and rabbit lungs: a comparative study. $\mathrm{Br} \mathrm{J}$ Radiol 2005;78:1018-27. https://doi.org/10.1259/bjr/13024611.

[10] Lewis RA, Yagi N, Kitchen MJ, Morgan MJ, Paganin D, Siu KKW, et al. Dynamic imaging of the lungs using x-ray phase contrast. Phys Med Biol 2005;50:5031-40. https://doi.org/10.1088/0031-9155/50/21/006.

[11] Mokso R, Schlepütz CM, Theidel G, Billich H, Schmid E, Celcer T, et al. GigaFRoST: the gigabit fast readout system for tomography. J Synchrotron Radiat 2017;24: 1250-9. https://doi.org/10.1107/s1600577517013522.

[12] Bayat S, Le DG, Porra L, Berruyer G, Nemoz C, Monfraix S, et al. Quantitative functional lung imaging with synchrotron radiation using inhaled xenon as contrast agent. Phys Med Biol 2001;46:3287-99. https://doi.org/10.1088/00319155/46/12/315.

[13] Thomlinson W, Elleaume H, Porra L, Suortti P. K-edge subtraction synchrotron Xray imaging in bio-medical research. Phys Medica 2018;49:58-76. https://doi.org/ 10.1016/j.ejmp.2018.04.389.

[14] Suortti P, Keyriläinen J, Thomlinson W. Analyser-based x-ray imaging for biomedical research. J Phys D Appl Phys 2013;46:494002. https://doi.org/ 10.1088/0022-3727/46/49/494002.

[15] Suortti P, Keyriläinen J, Thomlinson W. X-ray Phase-Contrast Mammography. Handb X-Ray. Imaging 2017:1025-48. https://doi.org/10.1201/9781351228251 52.

[16] Bonse U, Hart M. An X-ray interferometer. Appl Phys Lett 1965;6:155-6. https:// doi.org/10.1063/1.1754212.

[17] Momose A. Demonstration of phase-contrast X-ray computed tomography using an X-ray interferometer. Nucl Instruments Methods Phys Res Sect A Accel Spectrometers, Detect Assoc Equip 1995;352:622-8. https://doi.org/10.1016/ 0168-9002(95)90017-9.

[18] Diemoz PC, Bravin A, Coan P. Theoretical comparison of three X-ray phase-contrast imaging techniques: propagation-based imaging, analyzer-based imaging and grating interferometry. Opt Express 2012;20:2789. https://doi.org/10.1364/ oe.20.002789.

[19] Ando M, Sugiyama H, Maksimenko A, Rubenstein E, Roberson J, Shimao D, et al. X-ray dark-field imaging and its application to medicine. Radiat Phys Chem 2004; 71:899-904. https://doi.org/10.1016/j.radphyschem.2004.04.127.

[20] Burvall A, Lundström U, Takman PAC, Larsson DH, Hertz HM. Phase retrieval in X ray phase-contrast imaging suitable for tomography. Opt Express 2011;19:10359. https://doi.org/10.1364/oe.19.010359.

[21] Gureyev TE, Mayo SC, Nesterets YI, Mohammadi S, Lockie D, Menk RH, et al. Investigation of the imaging quality of synchrotron-based phase-contrast mammographic tomography. J Phys D Appl Phys 2014;47:365401. https://doi. org/10.1088/0022-3727/47/36/365401.

[22] Nesterets YI, Gureyev TE, Mayo SC, Stevenson AW, Thompson D, Brown JMC, et al A feasibility study of X-ray phase-contrast mammographic tomography at the Imaging and Medical beamline of the Australian Synchrotron. J Synchrotron Radiat 2015;22:1509-23. https://doi.org/10.1107/s160057751501766x.

[23] Leong AFT, Fouras A, Islam MS, Wallace MJ, Hooper SB, Kitchen MJ. High spatiotemporal resolution measurement of regional lung air volumes from $2 \mathrm{D}$ phase contrast x-ray images. Med Phys 2013;40:41909. https://doi.org/10.1118/ 1.4794926.

[24] Kitchen MJ, Buckley GA, Leong AFT, Carnibella RP, Fouras A, Wallace MJ, et al. Xray specks: low dose in vivo imaging of lung structure and function. Phys Med Biol 2015;60:7259. https://doi.org/10.1088/0031-9155/60/18/7259.

[25] Bayat S, Dullin C, Kitchen MJ, Lovric G. Synchrotron X-Ray-Based Functional and Anatomical Lung Imaging Techniques. Adv High-Resolution Tomogr Regen Med 2018:151-67. https://doi.org/10.1007/978-3-030-00368-5_10.

[26] Morgan KS, Parsons D, Cmielewski P, McCarron A, Gradl R, Farrow N, et al. Methods for dynamic synchrotron X-ray respiratory imaging in live animals. J Synchrotron Radiat 2020;27:164-75. https://doi.org/10.1107/ s1600577519014863.

[27] Hubbell JH, Seltzer SM. Tables of X-ray mass attenuation coefficients and mass energy-absorption coefficients $1 \mathrm{keV}$ to $20 \mathrm{MeV}$ for elements $\mathrm{Z}=1$ to 92 and 48 additional substances of dosimetric interest. Gaithersburg, MD, United States: National Inst. of Standards and Technology-PL; 1995.

[28] Jacobson B. Dichromatic Absorption Radiography. Dichromography. Acta Radiol 1953;39:437-52. https://doi.org/10.3109/00016925309136730.

[29] Mistretta CA, Ort MG, Kelcz F, Cameron JR, Siedband MP, Crummy AB. Absorption Edge Fluoroscopy Using Quasi-monoenergetic X-ray Beams*. Invest Radiol 1973;8: 402-12. https://doi.org/10.1097/00004424-197311000-00006.

[30] Rutt BK, Cunningham IA, Fenster A. Selective iodine imaging using lanthanum $\mathrm{K}$ fluorescence. Med Phys 1983;10:801-8. https://doi.org/10.1118/1.595447.

[31] Zhong Z, Chapman D, Menk R, Richardson J, Theophanis S, Thomlinson W. Monochromatic energy-subtraction radiography using a rotating anode source and a bent Laue monochromator. Phys Med Biol 1997;42:1751-62. https://doi.org/ 10.1088/0031-9155/42/9/007.

[32] Suortti P, Thomlinson W. A bent laue crystal monochromator for angiography at the NSLS. Nucl Instruments Methods Phys Res Sect A Accel Spectrometers, Detect Assoc Equip 1988;269:639-48. https://doi.org/10.1016/0168-9002(88)90145-3.

[33] Suortti P, Thomlinson W, Chapman D, Gmür N, Siddons DP, Schulze C. A single crystal bent Laue monochromator for coronary angiography. Nucl Instruments Methods Phys Res Sect A Accel Spectrometers, Detect Assoc Equip 1993;336: 304-9. https://doi.org/10.1016/0168-9002(93)91114-3.

[34] Riederer SJ, Mistretta CA. Selective iodine imaging using K-edge energies in computerized x-ray tomography. Med Phys 1977;4:474-81. https://doi.org/ 10.1118/1.594357.
[35] Elleaume H, Charvet AM, Corde S, Estève F, Le Bas JF. Performance of computed tomography for contrast agent concentration measurements with monochromatic x-ray beams: Comparison of K-edge versus temporal subtraction. Phys Med Biol 2002;47:3369-85. https://doi.org/10.1088/0031-9155/47/18/307.

[36] Marcucci C, Nyhan D, Simon BA. Distribution of pulmonary ventilation using Xeenhanced computed tomography in prone and supine dogs. J Appl Physiol 2001; 90:421-30. https://doi.org/10.1152/jappl.2001.90.2.421.

[37] Gefter WB, Functional CT. Imaging of the Lungs. Acad Radiol 2002;9:127-9. https://doi.org/10.1016/s1076-6332(03)80162-9.

[38] Kauczor H-U. Hyperpolarized Helium-3 Gas Magnetic Resonance Imaging of the Lung. Top Magn Reson Imaging 2003;14:223-30. https://doi.org/10.1097/ 00002142-200306000-00002.

[39] Castillo-Saldana D, Hague CJ, Coxson HO, Ryerson CJ. Using quantitative computed tomographic imaging to understand chronic obstructive pulmonary disease and fibrotic interstitial lung disease: state of the art and future directions. J Thorac Imaging 2020. https://doi.org/10.1097/RTI.0000000000000440.

[40] Fuld MK, Halaweish AF, Haynes SE, Divekar AA, Guo J, Hoffman EA. Pulmonary perfused blood volume with dual-energy CT as surrogate for pulmonary perfusion assessed with dynamic multidetector CT. Radiology 2013;267:747-56. https://doi. org/10.1148/radiol.12112789.

[41] Hoffman EA, Lynch DA, Barr RG, van Beek EJR, Parraga G, Investigators I. Pulmonary CT and MRI phenotypes that help explain chronic pulmonary obstruction disease pathophysiology and outcomes. J Magn Reson Imaging 2016; 43:544-57. https://doi.org/10.1002/jmri.25010.

[42] Giacomini JC, Gordon H, O'Neil R, Van Kessel A, Cason B, Chapman D, et al. Bronchial imaging in humans using xenon K-edge dichromography. Nucl Instruments Methods Phys Res Sect A Accel Spectrometers, Detect Assoc Equip 1998;406:473-8. https://doi.org/10.1016/s0168-9002(98)00195-8.

[43] Verbanck S, Paiva M. Gas mixing in the airways and airspaces. Compr Physiol 2011;1:809-34. https://doi.org/10.1002/cphy.c100018.

[44] Bayat S, Porra L, Suhonen H, Nemoz C, Suortti P, Sovijärvi ARA. Differences in the time course of proximal and distal airway response to inhaled histamine studied by synchrotron radiation CT. J Appl Physiol 2006;100. https://doi.org/10.1152/ japplphysiol.00594.2005.

[45] Layachi S, Rogerieux F, Robidel F, Lacroix G, Correction BS. Effet of combined nitrogen dioxide and carbon nanoparticle exposure on lung function during ovalbumin sensitization in Brown Norway rat (PLOS ONE). PLoS ONE 2013;8. https://doi.org/10.1371/annotation/d271d9c1-5588-4b43-85c3-d3de58ab61a4.

[46] Zhu Y, Samadi N, Martinson M, Bassey B, Wei Z, Belev G, et al. Spectral K-edge subtraction imaging. Phys Med Biol 2014;59:2485-503. https://doi.org/10.1088/ 0031-9155/59/10/2485.

[47] Bassey B, Martinson M, Samadi N, Belev G, Karanfil C, Qi P, et al. Multiple energy synchrotron biomedical imaging system. Phys Med Biol 2016;61:8180-98. https:// doi.org/10.1088/0031-9155/61/23/8180.

[48] Suhonen H, Porra L, Bayat S, Sovijärvi ARA, Suortti P. Simultaneous in vivo synchrotron radiation computed tomography of regional ventilation and blood volume in rabbit lung using combined K-edge and temporal subtraction. Phys Med Biol 2008;53. https://doi.org/10.1088/0031-9155/53/3/016.

[49] Jacquet M. High intensity compact Compton X-ray sources: Challenges and potential of applications. Nucl Instruments Methods Phys Res Sect B Beam Interact with Mater Atoms 2014;331:1-5. https://doi.org/10.1016/j.nimb.2013.10.078.

[50] Home - Lyncean Technologies, Inc. n.d. https://ynceantech.com/ (accessed June 6, 2020).

[51] Bech M, Bunk O, David C, Ruth R, Rifkin J, Loewen R, et al. Hard X-ray phasecontrast imaging with the Compact Light Source based on inverse Compton X-rays. J Synchrotron Radiat 2008;16:43-7. https://doi.org/10.1107/ s090904950803464x.

[52] Gradl R, Dierolf M, Hehn L, Günther B, Yildirim AÖ, Gleich B, et al. Propagationbased Phase-Contrast X-ray Imaging at a Compact Light Source. Sci Rep 2017;7. https://doi.org/10.1038/s41598-017-04739-w.

[53] Günther B, Dierolf M, Gradl R, Eggl E, Jud C, Hehn L, et al. The Munich Compact Light Source: Biomedical Research At a Laboratory-Scale Inverse-Compton Synchrotron X-ray Source. Microsc Microanal 2018;24:984-5. https://doi.org/ 10.1017/S143192761800541X.

[54] Kulpe S, Dierolf M, Braig E, Günther B, Achterhold K, Gleich B, et al. K-edge subtraction imaging for coronary angiography with a compact synchrotron X-ray source. PLoS ONE 2018;13. https://doi.org/10.1371/journal.pone.0208446.

[55] Jacquet M, Suortti P. Radiation therapy at compact Compton sources. Phys Medica 2015;31:596-600. https://doi.org/10.1016/j.ejmp.2015.02.010.

[56] Yagi N, Suzuki Y, Umetani K, Kohmura Y, Yamasaki K. Refraction-enhanced x-ray imaging of mouse lung using synchrotron radiation source. Med Phys 1999;26: 2190-3. https://doi.org/10.1118/1.598735.

[57] Sera T, Yokota H, Uesugi K, Yagi N. Airway distension during lung inflation in healthy and allergic-sensitised mice in vivo. Respir Physiol Neurobiol 2013;185: 639-46. https://doi.org/10.1016/j.resp.2012.12.002.

[58] Sznitman J. Respiratory microflows in the pulmonary acinus. J Biomech 2013;46: 284-98. https://doi.org/10.1016/j.jbiomech.2012.10.028.

[59] Sera T, Uesugi K, Yagi N, Yokota H. Numerical simulation of airflow and microparticle deposition in a synchrotron micro-CT-based pulmonary acinus model. Comput Methods Biomech Biomed Engin 2014;18:1427-35. https://doi. org/10.1080/10255842.2014.915030.

[60] Kitchen MJ, Lewis RA, Morgan MJ, Wallace MJ, Siew ML, Siu KKW, et al. Dynamic measures of regional lung air volume using phase contrast x-ray imaging. Phys Med Biol 2008;53:6065-77. https://doi.org/10.1088/0031-9155/53/21/012.

[61] Broche L, Perchiazzi G, Porra L, Tannoia A, Pellegrini M, Derosa S, et al. Dynamic Mechanical Interactions between Neighboring Airspaces Determine Cyclic Opening 
and Closure in Injured Lung. Crit Care Med 2017;45:687-94. https://doi.org/ 10.1097/CCM.0000000000002234.

[62] Broche L, Pisa P, Porra L, Degrugilliers L, Bravin A, Pellegrini M, et al. Individual airway closure characterized in vivo by phase-contrast CT imaging in injured rabbit lung. Crit Care Med 2019;47. https://doi.org/10.1097/ CCM.0000000000003838.

[63] Scaramuzzo G, Broche L, Pellegrini M, Porra L, Derosa S, Tannoia AP, et al. The Effect of Positive End-Expiratory Pressure on Lung Micromechanics Assessed by Synchrotron Radiation Computed Tomography in an Animal Model of ARDS. J Clin Med 2019;8:1117. https://doi.org/10.3390/jcm8081117.

[64] Scaramuzzo G, Broche L, Pellegrini M, Porra L, Derosa S, Tannoia AP, et al. Regional Behavior of Airspaces During Positive Pressure Reduction Assessed by Synchrotron Radiation Computed Tomography. Front Physiol 2019;10. https:// doi.org/10.3389/fphys.2019.00719.

[65] Lovric G, Mokso R, Schlepütz CM, Stampanoni M. A multi-purpose imaging endstation for high-resolution micrometer-scaled sub-second tomography. Phys Medica 2016;32:1771-8. https://doi.org/10.1016/j.ejmp.2016.08.012.

[66] Fardin L, Broche L, Lovric G, Larsson A, Bravin A, Bayat S. Mapping cardiacinduced lung motion using high-resolution time-resolved phase-contrast synchrotron computed tomography. Eur Respir J 2018;52.

[67] Bayat S, Fardin L, Mittone A, Larsson AS, Bravin A. Dynamic In Vivo Synchrotron Phase-Contrast X-Ray Lung Microscopy. Underst. lung Inj. from gene to Dysfunct., American Thoracic Society; 2020, p. A4102-A4102.

[68] Lovric G, Broche L, Schleputz CM, Fardin L, Schittny JC, Larsson AS, et al. Spatial Distribution of Ventilator Induced Lung Injury at the $\mu \mathrm{m}-\mathrm{Scale}$. An In-Vivo Synchrotron Phase-Contrast Microscopy Study in BALB/c Mice. D105. Crit. Care Vent. Induc. lung Inj. ARDS-from mice to biomarkers ARDS, American Thoracic Society; 2018, p. A7526-A7526.

[69] Bayat S, Strengell S, Porra L, Janosi TZ, Petak F, Suhonen H, et al. Methacholine and Ovalbumin Challenges Assessed by Forced Oscillations and Synchrotron Lung Imaging. Am J Respir Crit Care Med 2009;180:296-303. https://doi.org/10.1164/ rccm.200808-12110C.

[70] Porra L, Peták F, Strengell S, Neitola K, Janosi TZ, Suhonen H, et al. Acute cigarette smoke inhalation blunts lung responsiveness to methacholine and allergen in rabbit: Differentiation of central and peripheral effects. Am J Physiol - Lung Cell Mol Physiol 2010;299. https://doi.org/10.1152/ajplung.00033.2010.

[71] Bayat S, Porra L, Suhonen H, Suortti P, Sovijärvi ARA. Paradoxical conducting airway responses and heterogeneous regional ventilation after histamine inhalation in rabbit studied by synchrotron radiation CT. J Appl Physiol 2009;106. https://doi.org/10.1152/japplphysiol.90550.2008.

[72] Winkler T, Venegas JG. Complex airway behavior and paradoxical responses to bronchoprovocation. J Appl Physiol 2007;103:655-63. https://doi.org/10.1152/ japplphysiol.00041.2007.

[73] Hantos Z, Daroczy B, Suki B, Nagy S, Fredberg JJ. Input impedance and peripheral inhomogeneity of dog lungs. J Appl Physiol 1992. https://doi.org/10.1152/ jappl.1992.72.1.168.

[74] Porra L, Dégrugilliers L, Broche L, Albu G, Strengell S, Suhonen H, et al. Quantitative Imaging of Regional Aerosol Deposition, Lung Ventilation and Morphology by Synchrotron Radiation CT. Sci Rep 2018;8. https://doi.org/ 10.1038/s41598-018-20986-x.
[75] Bayat S, Porra L, Albu G, Suhonen H, Strengell S, Suortti P, et al. Effect of positive end-expiratory pressure on regional ventilation distribution during mechanical ventilation after surfactant depletion. Anesthesiology 2013;119. https://doi.org/ 10.1097/ALN.0b013e318291c165.

[76] Mead J, Takishima T, Leith D. Stress distribution in lungs: a model of pulmonary elasticity. J Appl Physiol 1970;28:596-608. https://doi.org/10.1152/ jappl.1970.28.5.596.

[77] Bayat S, Porra L, Broche L, Albu G, Malaspinas I, Doras C, et al. Effect of surfactant on regional lung function in an experimental model of respiratory distress syndrome in rabbit. J Appl Physiol 2015;119:290-8. https://doi.org/10.1152/ japplphysiol.00047.2015.

[78] Porra L, Bayat S, Malaspinas I, Albu G, Doras C, Broche L, et al. Pressure-regulated volume control vs. volume control ventilation in healthy and injured rabbit lung: An experimental study. Eur J Anaesthesiol 2016;33. https://doi.org/10.1097/ EJA.0000000000000485.

[79] Porra L, Broche L, Dégrugilliers L, Albu G, Malaspinas I, Doras C, et al. Synchrotron Imaging Shows Effect of Ventilator Settings on Intrabreath Cyclic Changes in Pulmonary Blood Volume. Am J Respir Cell Mol Biol 2017;57:459-67. https://doi. org $/ 10.1165 / \mathrm{rcmb} .2017-0007$ oc.

[80] Brombal L, Arfelli F, Delogu P, Donato S, Mettivier G, Michielsen K, et al. Image quality comparison between a phase-contrast synchrotron radiation breast CT and a clinical breast CT: a phantom based study. Sci Rep 2019;9. https://doi.org/ 10.1038/s41598-019-54131-z.

[81] Tavakoli Taba S, Baran P, Nesterets YI, Pacile S, Wienbeck S, Dullin C, et al. Comparison of propagation-based CT using synchrotron radiation and conventional cone-beam CT for breast imaging. Eur Radiol 2020;30:2740-50. https://doi.org/10.1007/s00330-019-06567-0.

[82] Gromann LB, De Marco F, Willer K, Noël PB, Scherer K, Renger B, et al. In-vivo Xray Dark-Field Chest Radiography of a Pig. Sci Rep 2017;7. https://doi.org/ 10.1038/s41598-017-05101-w.

[83] Zanette I, Zdora M-C, Zhou T, Burvall A, Larsson DH, Thibault P, et al. X-ray microtomography using correlation of near-field speckles for material characterization. Proc Natl Acad Sci 2015;112:12569-73. https://doi.org/ 10.1073/pnas.1502828112.

[84] Labriet H, Berujon S, Broche L, Fayard B, Bohic S, Stephanov O, et al. 3D histopathology speckle phase contrast imaging: From synchrotron to conventional sources. Prog. Biomed. Opt. Imaging - Proc. SPIE, vol. 10948, 2019. Doi: 10.1117/ 12.2511944.

[85] Strengell S, Keyriläinen J, Suortti P, Bayat S, Sovijärvi ARA, Porra L. Radiation dose and image quality in K-edge subtraction computed tomography of lung in vivo. J Synchrotron Radiat 2014;21. https://doi.org/10.1107/S160057751401697X.

[86] McCollough CH, Leng S, Yu L, Fletcher JG. Dual-and multi-energy CT: principles, technical approaches, and clinical applications. Radiology 2015;276:637-53. https://doi.org/10.1148/radiol.2015142631.

[87] Kong X, Sheng HX, Lu GM, Meinel FG, Dyer KT, Schoepf UJ, et al. Xenon-enhanced dual-energy CT lung ventilation imaging: techniques and clinical applications. Am J Roentgenol 2014;202:309-17. https://doi.org/10.2214/AJR.13.11191.

[88] García-Moreno F, Kamm PH, Neu TR, Bülk F, Mokso R, Schlepütz CM, et al. Using X-ray tomoscopy to explore the dynamics of foaming metal. Nat Commun 2019;10: 1-9. https://doi.org/10.1038/s41467-019-11521-1. 